\title{
A versatile toolkit for CRISPR-Cas13-based RNA manipulation in Drosophila
}

\author{
Nhan Huynh, Noah Depner, Raegan Larson and Kirst King-Jones*
}

\author{
* Correspondence: kirst.king-jones@ \\ ualberta.ca \\ Department of Biological Sciences, \\ University of Alberta, G-504 \\ Biological Sciences Bldg., \\ Edmonton, Alberta T6G 2E9, Canada
}

\begin{abstract}
Advances in CRISPR technology have immensely improved our ability to manipulate nucleic acids, and the recent discovery of the RNA-targeting endonuclease Cas13 adds even further functionality. Here, we show that Cas 13 works efficiently in Drosophila, both ex vivo and in vivo. We test 44 different Cas 13 variants to identify enzymes with the best overall performance and show that Cas 13 could target endogenous Drosophila transcripts in vivo with high efficiency and specificity. We also develop Cas13 applications to edit mRNAs and target mitochondrial transcripts. Our vector collection represents a versatile tool collection to manipulate gene expression at the post-transcriptional level.
\end{abstract}

Keywords: CRISPR, Cas13, CasRX, Drosophila, RNA manipulation, crRNA design

\section{Background}

Most bacterial and archaeal genomes harbor clustered regularly interspaced short $p$ alindromic repeats (CRISPR) and encode CRISPR-associated proteins (Cas) as a defense system against bacteriophages and other invading nucleic acids [1-3]. The immune response of all CRISPR/Cas systems characterized to date includes three steps: (i) adaptation and spacer acquisition, where a piece of the invading genome is incorporated into the CRISPR array, (ii) the expression of mature CRISPR-RNAs (gRNAs) from the processed CRISPR array, and (iii) interference, where Cas enzymes are guided by the gRNAs to the corresponding region of the invading genome for cleavage and degradation $[4,5]$. The CRISPR/Cas class II systems use a single, multidomain Cas effector protein [6]. Because of its simplicity, the single multidomain effector found in class II organisms is used in current CRISPR methods. Class II type II CRISPR Cas9 was one of the first Cas proteins studied in detail, which led to its widespread use for genomic engineering (Fig. 1a) [6-11]. Currently, CRISPR/Cas9 approaches allow scientists to precisely alter gene function via (i) classic CRISPR to introduce short INDELs, (ii) HRbased CRISPR for homology-based gene replacements or deletions, (iii) somatic CRIS PR for conditional gene disruption, (iv) CRISPRi, ( $\mathrm{i}=$ interference) to interfere with gene transcription, and (v) CRISPRa ( $\mathrm{a}=$ activation) to upregulate gene activity. Studies have shown that it is possible to conditionally target genes of interest by exerting

(c) The Author(s). 2020 Open Access This article is licensed under a Creative Commons Attribution 4.0 International License, which permits use, sharing, adaptation, distribution and reproduction in any medium or format, as long as you give appropriate credit to the original author(s) and the source, provide a link to the Creative Commons licence, and indicate if changes were made. The images or other third party material in this article are included in the article's Creative Commons licence, unless indicated otherwise in a credit line to the material. If material is not included in the article's Creative Commons licence and your intended use is not permitted by statutory regulation or exceeds the permitted use, you will need to obtain permission directly from the copyright holder. To view a copy of this licence, visit http:/creativecommons.org/licenses/by/4.0/. The Creative Commons Public Domain Dedication waiver (http://creativecommons.org/publicdomain/zero/1.0/) applies to the data made available in this article, unless otherwise stated in a credit line to the data. 


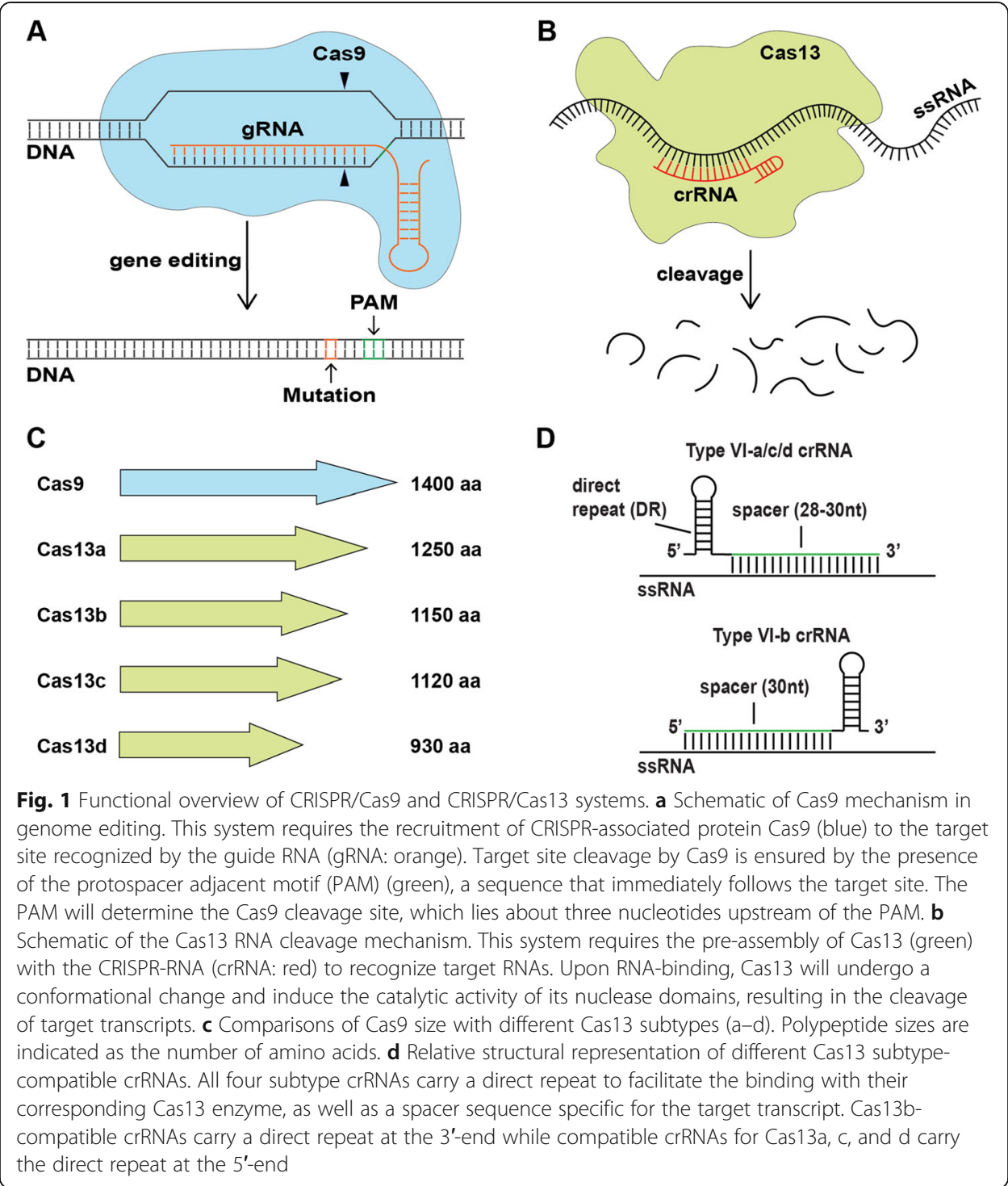

spatial and temporal control over Cas9 expression or using ligand-activated Cas9 variants $[8,10,12,13]$. The rapid advances in CRISPR technologies have made it a popular choice over earlier nuclease-based gene-editing approaches like meganucleases (MNs) $[14,15]$, zinc finger nucleases (ZFNs) [16-18], and transcription activator-like effector nucleases (TALENs) $[19,20]$.

The recent introduction of the class II type VI CRISPR/Cas13 system further expands the existing technology in significant ways. Like Cas9, Cas13 uses a guide RNA (CRIS PR-RNA, aka crRNA) to identify its substrate, which is RNA rather than DNA (Fig. 1b). Cas13 enzymes have two distinct catalytic activities: (i) an RNAse activity that is mediated by two higher eukaryotic and prokaryotic nucleotide (HEPN)-binding domains and (ii) a gRNA maturation activity, possibly a combination of activities located in the HEPN2 and Helical-1 domains [21, 22]. There are currently four subtypes identified in the Cas13 family, including Cas13a (aka C2c2), Cas13b, Cas13c, and Cas13d. All Cas13 family members are smaller than Cas9, with Cas13d being the smallest protein. The small size of Cas13 proteins makes them suitable for molecular genetics (Fig. 1c). All 
Cas13 enzymes require a 60-66-nucleotide-long crRNA to ensure target specificity [2, 3 , 23]. Similar to the gRNA in the CRISPR/Cas9 system, the crRNA used by Cas13 forms a short hairpin structure next to a short spacer sequence (28-30 nucleotides) that is specific to the target transcript (Fig. 1d). Since CRISPR/Cas13 mediates RNA degradation, it holds the promise to replace or complement RNA interference (RNAi) approaches or other systems that interfere with transcript levels, such as CRISPRi. Despite being a powerful tool, RNAi often suffers from low efficiencies or off-target effects, whereas Cas9-based CRISPRi requires a protospacer adjacent motif (PAM), thus limiting the flexibility by which target sequences can be selected [24-28]. It is desirable to examine whether CRISPR/Cas13 can offer better specificity and efficiency than these other interference techniques.

Drosophila melanogaster is a versatile genetic model organism that is used to study a wide variety of biological processes. Traditional techniques to analyze gene function in Drosophila include the generation of mutations via chemical mutagens and transposable P-elements, or the use of transgenes to trigger RNAi and to express cDNAs for gain-of-function studies via the Gal4/UAS system [29-33]. Like other model organisms, the CRISPR/Cas9 endonucleases have been quickly adopted by Drosophila researchers [10, 24, 25, 34-39]. CRISPR-based techniques are remarkably precise and, therefore, ideal for replacing, validating, and complementing traditional approaches, in particular procedures relying on the expression of RNAi or cDNA transgenes [40, 41]. Also, the large worldwide collection of gRNAs stocks has ensured the quick adaptation of CRIS PR/Cas9 into mainstream Drosophila research [6, 42, 43]. Given the potential of CRIS PR/Cas13-based methods to replace current techniques, we explored its feasibility and reliability in Drosophila.

Our lab studies signaling pathways that control ecdysone and heme biosynthesis in the larval prothoracic gland (PG), which is part of a larger structure called the ring gland. The PG is a popular model for investigating fundamental aspects of insect endocrinology and allows for the study of external cues that control the timing of ecdysone pulses [44]. Recently, we carried out a genome-wide PG-specific RNAi screen that identified 1906 genes with critical roles in larval development [45]. In follow-up experiments, however, we often were unable to validate the RNAi-induced phenotypes by independent RNAi lines, either because no such lines existed or because other RNAi lines did not replicate the phenotype. This prompted us to develop CRISPR-based methods that could validate the RNAi results by an unrelated methodology. We previously generated two CRISPR/Cas9 toolkit collections and could use them to validate some RNAi phenotypes. However, specific issues still exist, including inconsistent gRNA efficiency and early lethality. We sought to investigate the possibility of adapting the CRISPR/Cas13 system for interference and other potential applications of this system in Drosophila melanogaster.

We generated and evaluated the catalytic activity of Drosophila codon-optimized Cas13 (a-d) variants in a cell line derived from Sg4 embryonic cells. We refer to these Cas13 variants as CasFA[n], CasFB[n], CasFC $[n]$, and CasFX[n], respectively $(\mathrm{F}=$ fruit fly, A-C indicates the Cas13 subfamily, CasFX is the fly version of CasRX, and [n] indicates variant number) (Fig. 2a-d). "CasRX" was coined by Konermann et al. for the Cas13d ortholog isolated from Ruminococcus flavefaciens XPD3002 to distinguish it from other Cas13d variants [46]. Since we generated fly-optimized versions of CasRX, 


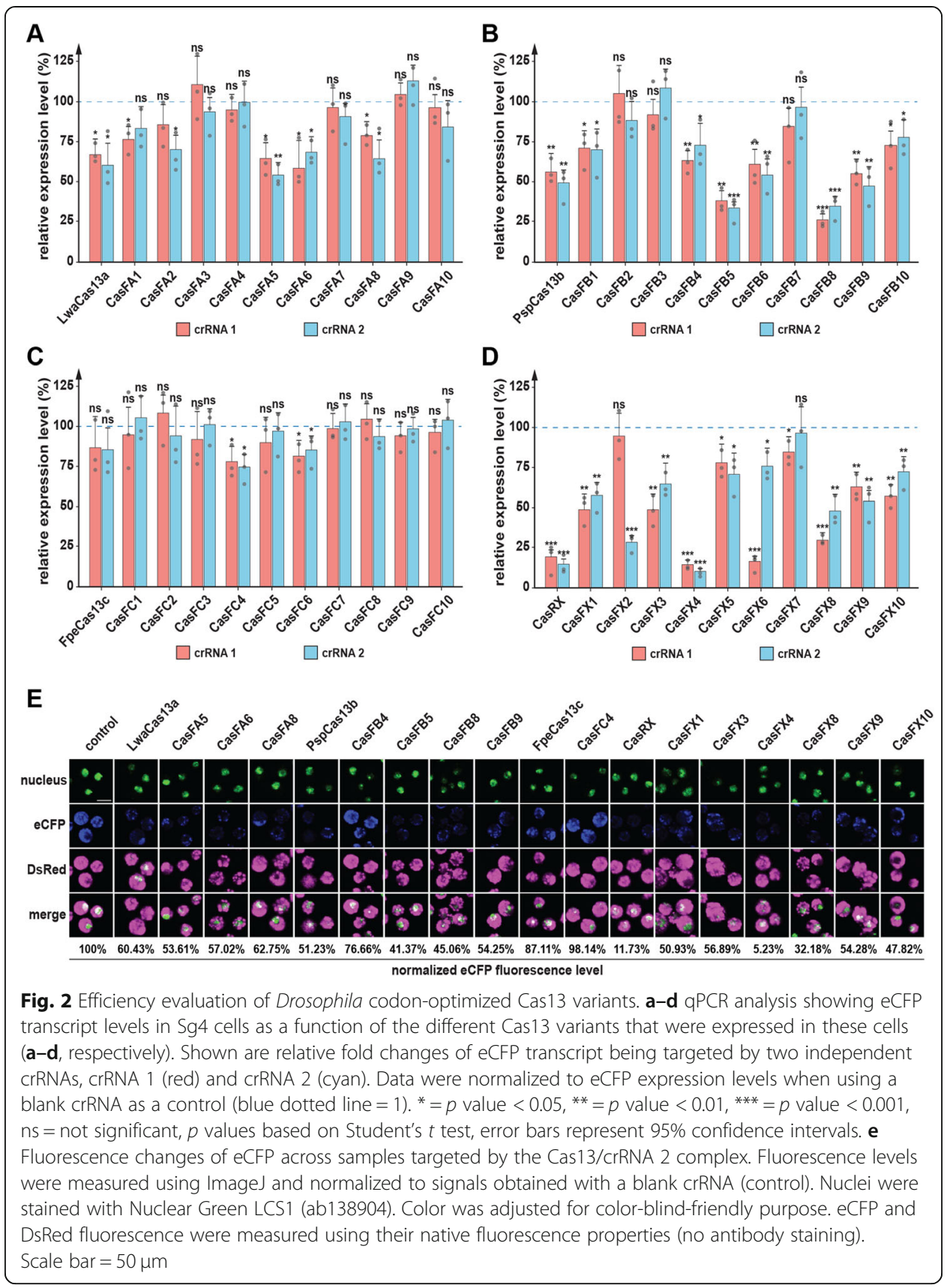

we refer to these versions as CasFX. Once we had identified a fly-optimized Cas13 variant, we used this variant to adapt existing Cas13 mammalian cell culture applications for Drosophila cells, such as transcript tracking and RNA modification [23, 47-52]. These ex vivo procedures formed the basis for generating a collection of transgenic CRISPR/Cas13 tools designed for in vivo RNA targeting. In particular, we generated four Cas13 transgenic lines, namely two that either ubiquitously express CasFB or CasFX, and two that express either CasFB or CasFX under UAS control. The UAS lines allow tissue-specific expression of CasFX and CasFB by crossing them to Gal4expressing flies. As proof-of-principle that these Cas13 transgenes work effectively 
in vivo, we generated seven crRNA transgenes to target three genes we are studying in our lab.

\section{Results}

\section{Generation and characterization of Drosophila-optimized Cas13 enzymes}

We generated ten Cas13 variants for each of the four Cas13 family members (a-d) by optimizing different codon subsets for codon usage in Drosophila. Specifically, we made ten constructs based on the Leptotrichia wadei Cas13a gene (LwaCas13a), ten variants based on the Prevotella sp. P5-125 Cas13b gene (PspCas13b), ten versions based on the Fusobacterium perfoetens Cas13c gene (FpeCas13c), and ten forms of the Ruminococcus flavefaciens XPD3002 Cas13d gene (aka CasRX) (Additional file 2: Table S1). We chose these Cas13 orthologs for the following reasons: (i) based on studies in mammalian and plant cells, LwaCas13a, PspCas13b, and CasRX showed improved and robust catalytic efficiency when compared to other Cas13 orthologs [23, 46, 50, 53], (ii) unlike some Cas13 orthologs, the Cas13 genes we chose for our studies do not require a specific protospacer flanking sequence (PFS) for efficient target RNA identification [23, 46, 50, 53]. In the case of PspCas13b, the original study, which was performed in Escherichia coli, showed that the PFS is necessary for RNA cleavage activity. However, when the same enzyme was tested in mammalian cells and plants, the PFS was no longer required [48, 49, 52]. Finally, (iii) we also selected Cas13c, since only a few studies have examined this Cas13 subtype [23].

To evaluate the RNA degradation efficiency of these fruit fly-optimized Cas13 enzymes, we needed to establish a stable reporter gene cell line. For this, we used the PhiC31 integrase system to generate a dual-reporter transgene in the Drosophila embryo cell line Sg4-PP-27F [54] that simultaneously expressed eCFP (enhanced Cyan Fluorescent Protein) and DsRed (Discosoma Red fluorescent protein) (Additional file 1: Fig. S1A, C). Sg4 is one of four embryonic cell lines isolated from the original Schneider's line 2 (S2) and differs from the popular S2 cells in a range of transcriptional properties [55]. Importantly, Sg4-PP-27F cells were modified from the original Sg4 cells by adding a PhiC31 docking site to the second chromosome [54]. The inserted eCFP and DsRed transgenes are each controlled by the ubiquitous actin $5 \mathrm{C}$ promoter (act5C). To ensure this transgene's stability, we added a $\mathrm{Neo}^{R}$ gene cassette, which encodes aminoglycoside kinase and ensures cell survival in the presence of G418 antibiotics [56]. We refer to this new transgenic cell line as Sg4_CD $(\mathrm{C}=\mathrm{eCFP}, \mathrm{D}=\mathrm{DsRed})$, and our subsequent cell culture experiments were based on this line. To transform the Sg4_CD cell line with appropriate vectors, we generated plasmids that harbored a single copy of a given Cas13 variant and a single crRNA (the vector allows for adding multiple crRNAs). These constructs, here referred to as $\mathrm{pC} 13 \mathrm{cr} 01$, allowed us to simultaneously express Cas13 as well as its crRNA in transfected cells (Additional file 1: Fig. S1D, Additional file 3: Table S2). To ensure stable transfection, we also included the PURO gene in the $\mathrm{pC} 13 \mathrm{cr} 01$ vector. The PURO gene encodes the puromycin Nacetyltransferase, which allows cells to survive in media supplemented with puromycin [57, 58] (Additional file 1: Fig. S1D, Additional file 3: Table S2). Thus, the presence of two resistance markers allowed for dual selection during the transfection experiments. Besides testing the Drosophila-optimized Cas13 variants, we also examined the 
efficiency of the original Cas13 orthologs in the Sg4_CD cell line (Additional file 2: Tables S1, Additional file 3: Table S2).

We measured the efficiency of the Cas13 variants by targeting one of the two reporter gene mRNAs and quantifying mRNA levels via qPCR. To accomplish this, for each Cas13 variant, we used two independent single crRNAs targeting eCFP mRNA (crRNA1 and crRNA2, Fig. 2), while the DsRed mRNA was not targeted and served as a control (Additional file 1: Fig. S2, Additional file 4: Table S3). To ensure that any observed differences derived only from the catalytic activity of the Cas13/crRNA complex, and not from either Cas13 or the crRNA itself, we also tested the eCFP expression level in the presence of a non-targeting (NT) Cas13/crRNA complex. In our hands, the different Cas13 variants showed a wide range of RNA-targeting efficiency, with some of the variants failing to trigger RNA degradation. The original Cas13a (aka LwaCas13a) showed roughly 35-40\% eCFP knock-down efficiency, while the best-performing Drosophila variant, CasFA5, was only slightly better and exhibited $47 \%$ efficiency (Fig. 2a). For the Cas13b (aka PspCas13b) variants, we measured 45-51\% efficiency for the original Cas13b enzyme, while the best-performing Drosophila variants were CasFB5 and CasFB8, both of which were $65-70 \%$ efficient (Fig. 2b). The Cas13c group was the least efficient in knocking down eCFP, with the best line, CasFC4, only accomplishing a $25 \%$ knock-down (Fig. 2c). In contrast, the Cas13d group performed best, displaying $82 \%$ efficiency for the original Cas13d (CasRX) enzyme, whereas the CasFX4 variant was even better and reached a 90\% knock-down (Fig. 2d).

To validate these qPCR data, we quantified the protein levels of eCFP and DsRed based on their fluorescence and Western blotting. We selected the best-performing enzyme variants from all four groups, namely three CasFA variants, four CasFB versions, one CasFC enzyme, and six CasFX forms. We then assessed the efficiency of the eCFP knock-down via immunofluorescence (Fig. 2e) and Western blotting (Additional file 1: Fig. S3A-D). Both approaches showed comparable results and confirmed that CasFX4 was the overall most efficient Cas13 enzyme of the entire cohort, showing 90\% and 95\% efficiency on the mRNA and protein levels, respectively.

Next, we sought to investigate whether the subcellular localization of Cas13 would affect the enzyme's catalytic activity. Since mRNAs mature in the nucleus but are translated in the cytoplasm, we wondered if Cas13 performance could be improved by identifying which cellular compartment is optimal for Cas13 activity. To test this, we selected the original Cas13 variants and their corresponding best-performing Drosophila counterparts (CasFA5, CasFB8, CasFC4, and CasFX4) and fused them either with a nuclear localization signal (NLS) or a nuclear export signal (NES) (Additional file 1: Fig. S3E). These constructs were based on similar designs from other studies and our approaches (Additional file 1: Fig. S3F) [8, 10, 12, 35, 39, 59-62]. Then, as described above, we again examined how efficiently eCFP was knocked down. Overall, we observed similar efficiencies when the same Cas13 variant was tested in the nucleus or cytoplasm, indicating that the catalytic activity of these Cas 13 variants was independent of the subcellular localization (Additional file 1: Fig. S3G). For LwaCas13a, PspCas13b, and CasRX, this result is consistent with a previous study in plants [52]. Since we found no significant differences, we decided to use Cas13 variants without any localization signal for experiments that followed. 
Together, these data suggested that the Cas13 variants retain their RNA-cleaving activity in Drosophila Sg4_CD cells, but efficiencies varied considerably. Among the Drosophila codon-optimized Cas13 enzymes we generated, we noticed consistent and robust efficiency of two CasFB versions (namely CasFB5 and CasFB8) and the overall best Cas13 variant, CasFX4.

\section{Evaluating the collateral activity of Drosophila-optimized Cas 13 variants}

Studies in Escherichia coli showed that once the Cas13/crRNA complex is bound to its target RNA, the HEPN-nuclease domains become active and are capable of cleaving not just the intended target, but also RNA molecules that are in the vicinity of the Cas13/RNA complex, resulting in the non-specific RNA degradation referred to as "collateral activity" (Additional file 1: Fig. S4A) [21-23, 63]. Subsequent studies reported that the collateral activity of Cas13 varied from system to system. While non-specific RNA degradation was detected in human U87 glioblastoma cells [63], no collateral activity was detected in human embryonic kidney 293FT cells or in the plant Nicotiana benthamiana [23, 49, 50]. To test for collateral activity in our hands, we examined the best-performing Cas13 variants using the same transgenic cell line Sg4_CD. Specifically, we co-expressed eCFP, DsRed, and $\mathrm{Neo}^{\mathrm{R}}$ independently, each with an act5C promoter. Since eCFP, DsRed, and aminoglycoside kinase (encoded by $N e o^{R}$ gene) are foreign genetic components, we reasoned that manipulating their expression via Cas13 would not have a significant impact on the physiology of SG4_CD cells. The idea was to target eCFP with specific crRNAs in the presence of Cas13 and monitor the expression of DsRed as a readout for collateral activity. Both eCFP and DsRed were presumed to be highly expressed in a coordinate fashion, since the act5C promoter controlled each transgene. As such, if the interference activity of Cas 13 was not specific to eCFP, we expected to detect differences in DsRed expression via qPCR. Using this approach, our data showed that the selected Cas13/crRNA complexes only affected target eCFP expression, while DsRed expression appeared unperturbed (Additional file 1: Fig. S4B). These data suggest that the tested Cas13 enzymes did not have any detectable collateral activity, at least not in the Drosophila Sg4_CD cell line.

\section{Testing the fidelity of Drosophila Cas13 variants}

Our efforts identified several Cas13 versions that efficiently degraded target RNAs in Drosophila cells while exhibiting no detectable collateral activity. Next, we wanted to assess how mismatches between crRNAs and their cognate target RNA would affect RNA degradation as a means to define Cas13 fidelity. In particular, we were curious as to whether Cas13 would display higher fidelity-and as such, lower off-target ratesthan RNA interference (RNAi), which is widely used in a variety of research models, ranging from cell culture to whole organisms [64-66]. While RNAi is an attractive and powerful tool, its usability is often hampered by its off-target activity, which can make it challenging to interpret phenotypes, and validation strategies involving codonmodified genes/cDNAs are cumbersome and harbor pitfalls [26, 67, 68]. Other validation strategies include non-overlapping RNAi constructs targeting distinct regions on the mRNA, classic mutants, or conditional CRISPR/Cas9 approaches. To test the propensity of our Cas13 enzymes to degrade off-target RNAs due to small sequence 
differences, we selected the six top-performing variants for which we had not detected any collateral activity (CasFA5, CasFB5, CasFB8, CasFC4, CasFX4, and CasFX8). Specifically, we generated mismatches in the crRNA-2 spacer sequence and measured the ability to degrade its target RNA, eCFP. To indicate the mismatch location, we referenced the position of the altered nucleotide relative to the stem loop-forming direct repeat of the crRNA. The nucleotide at position 1 represents the one closest to the DR, and the highest number corresponds to the nucleotide farthest away from the DR.

Among all variants that we tested, all had a central region that appeared to be intolerant to single mismatches. The CasFA5, CasFB5, and CasFB8 variants showed some tolerance to single mismatches outside the core region, namely nucleotides \#1-3 at the 5 '-end and nucleotides $\# 28$ and higher at the $33^{\prime}$-end. In contrast, the core region showed no tolerance to mismatches (Fig. 3a-d). Remarkably, CasFC4, CasFX4, and CasFX8 variants showed no tolerance for mismatches throughout the entire range, including the extreme $5^{\prime}$ and $3^{\prime}$ ends. To examine this further, we tested the outermost nucleotides for both CasFX variants (position \#1 and \#30). Even single mismatches at either end of the spacer region abrogated interference activity, indicating that these two variants are highly specific and have the lowest off-target potential (Fig. 3e, f). Since four of the variants had some tolerance towards a single mismatch, we further examined mismatch tolerance by introducing more than one mutation per crRNA. Specifically, we generated constructs encoding two, three, or four mismatches in the eCFPcrRNA. In all tested conditions, we included at least one mismatch from the extreme $5^{\prime}$ or $3^{\prime}$ end of the spacer. In our hands, none of the Drosophila Cas13 variants exhibited tolerance to crRNAs with mismatches of more than one nucleotide (Additional file 1: Fig. S4 C-H). These data are in agreement with other studies using similar approaches $[51,69,70]$. Taken together, this suggests that the Drosophila Cas13 variants tested here are highly specific and display no tolerance to a single mismatch in the core region of the spacer, and none of the enzymes were functional with two mismatches in the crRNA. The CasFA, CasFB5, and CasFB8 variants did tolerate a single mismatch located at either end outside the core region. In contrast, the CasFC4, CasFX4, and CasFX8 variants appeared to require a perfect match of the entire spacer region to mediate interference. We conclude that the CasFX4 and CasFX8 variants will likely have the lowest off-target rate while retaining optimal RNA-targeting efficiency among the Cas13 enzymes tested here.

\section{Nuclease-dead CasFX for applications involving transcript detection}

The CRISPR/Cas9 system has been modified to allow for non-nuclease activities, such as for transcription interference (CRISPRi) as well as transcriptional activation (CRIS PRa) $[8,10,12,39]$. Similarly, the Cas13 system can also be adapted for other purposes and may be more suitable for certain applications than CRISPR/Cas9-based methods. For instance, the ability to target RNA instead of DNA has the advantage that it is reversible. Also, Cas13 may allow for the development of techniques that cannot be accomplished by the corresponding CRISPR/Cas9 approaches: By abolishing the nuclease activity of Cas13 while retaining its RNA-binding capability, one could use the enzyme to specifically target RNAs to track these transcripts in the cell. Another option would be to fuse Cas13 with different protein domains to affect post-transcriptional 


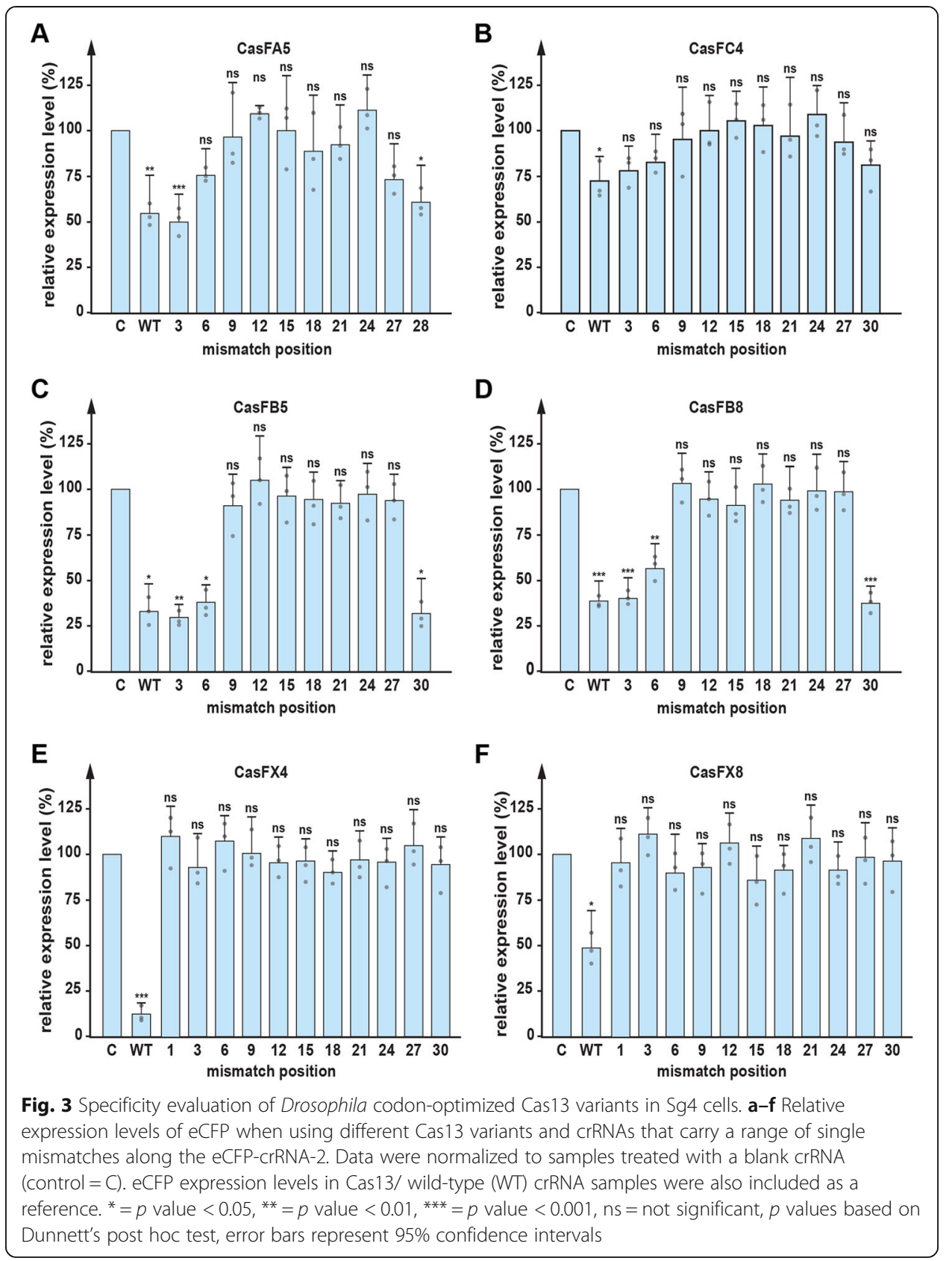

processing of target mRNAs, e.g., altering transcript splicing or stability. Specific efforts have been made to investigate these applications with promising results [48-50, 52, 71].

We were particularly interested in a nuclease-deficient Cas13 variant as a tool to validate specific RNA-protein interactions. For our proof-of-principle approach, we selected the Cas13 variant with the most consistent, robust, and specific interference activity, CasFX4 (hereafter referred to as simply CasFX), and introduced quadruple mutations in the catalytic HEPN domains (R239A/H244A/R858A/H863A). These mutations abolish the nuclease activity but not RNA-binding activity in the CasRX variant [50, 52, 71] (Fig. 4a). We first tested whether the mutant CasFX still retained nuclease 


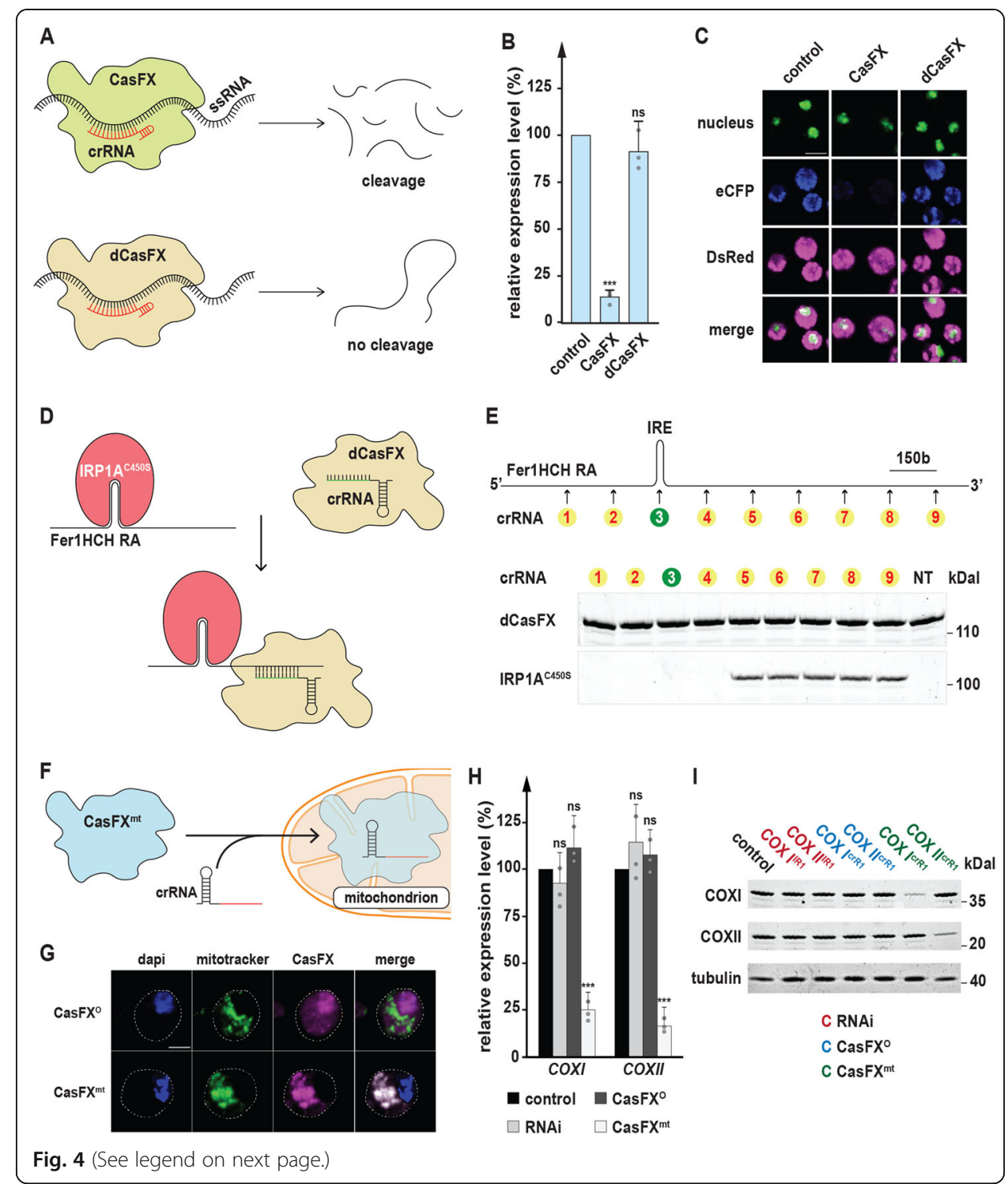


(See figure on previous page.)

Fig. 4 Properties of modified Cas13 variants. a Schematic of nuclease-dead CasFX (dCasFX) activity. dCasFX carries quadrupl e point mutations that abolish its nuclease activity. As a result, the dCasFX/crRNA complex can be recruited and bind to target transcripts, but it cannot cleave the RNA. $\mathbf{b}$ Evaluation of Cas 13 cleavage efficiency of dCasFX compared to wild-type CasFX. qPCR data represent expression levels of eCFP. Data were normalized to samples treated with blank crRNA (control). ${ }^{*}=p$ value $<0.05$, ${ }^{* *}=p$ value $<0.01$, ${ }^{* * *}=p$ value $<0.001, p$ values based on Student's $t$ test, error bars represent $95 \%$ confidence intervals. $\mathbf{c}$ eCFP fluorescence when targeted by either CasFX or dCasFX. Nuclei were stained with nuclear green DCS1 (Abcam ab138904). Color was adjusted for color-blind-friendly purpose. eCFP and DsRed fluorescence were measured using their native fluorescence property without using antibody staining. Scale bar $=50 \mu \mathrm{m}$. $\mathbf{d}$ Schematic of dCasFX for the validation of RNA-protein interactions. dCasFX and crRNA targeting Fer1 $\mathrm{HCH}-$ RA mRNA were transfected together in one sample. Fer1HCH-RA and IRP1A ${ }^{\mathrm{C} 450 \mathrm{~S}}$, a constitutively RNAbinding form of IRP1A that interacts with the iron-responsive element (IRE) in the Fer1HCH-RA mRNA, were transformed together in a different sample. The two samples were each lysed and combined, followed by immunoprecipitation (IP) of dCasFX (utilizing the attached HA tag) to test for the presence of IRP1A in the pull-down assay. e Western blot showing the IP of dCasFX combined with different crRNAs along Fer1HCH$R A$ mRNA and the detection of IRP1A in corresponding samples. $\mathbf{f}$ Functional schematic of CasFX that carries a mitochondrial localization signal (CasFX ${ }^{\mathrm{mt}}$ ). At the $N$ terminus, CasFX ${ }^{\mathrm{mt}}$ is fused with the $\operatorname{tim} 23$ mitochondrial signal sequence. Upon binding with crRNA, the complex will localize into mitochondria and target mitochondrial-encoded transcripts. g Mitochondrial localization of CasFX' ${ }^{\mathrm{mt}}$. Nuclei were stained with DAPI (blue) while mitochondria were stained with mitotracker green (Cell signaling 9074S) and CasFX polypeptide was stained with anti-HA antibody (magenta). Scale bar $=25 \mu \mathrm{m}$. Color was adjusted for colorblind-friendly purpose. $\mathbf{h}$ The relative expression level of mitochondrial-encoded transcripts, COXI and COXII, targeted by RNAi, CasFX ${ }^{\circ}$, and CasFXmt . Data were normalized to samples treated with no transfected plasmid (control). ${ }^{*}=p$ value $<0.05,{ }^{* *}=p$ value $<0.01,{ }^{* * *}=p$ value $<0.001$, ns $=$ not significant, $p$ values based on Dunnett's post hoc test, error bars represent $95 \%$ confidence intervals. i Western blotting of COXI and COXII when being targeted by RNAi, CasFX' and CasFX'

activity by testing our validated crRNAs against eCFP in the Sg4_CD cell line. As expected, the mutant CasFX failed to interfere with the expression level of eCFP, whereas the wild-type variant worked efficiently (Fig. 4b, c). We conclude that this mutant CasFX variant, similar to the corresponding variants in other species, lost its nuclease activity. We hereafter refer this variant as $\mathrm{dCasFX}(\mathrm{d}=\mathrm{dead})$.

To assess whether crRNA-guided dCasFX would specifically interact in a nondestructive manner with its intended target mRNA, we tested its ability to co-IP a protein known to bind to the same mRNA. As such, immunoprecipitation of dCasFX should pull down the mRNA as well as its bound protein, which can be detected via Western blotting. This approach is useful to validate the RNA-binding activity of $\mathrm{dCasFX}$, as well as the interaction between mRNA and the interrogated protein. To test this, we used an isoform of the ferritin heavy chain 1 mRNA (Fer1HCH-RA), which carries a canonical iron-responsive element (IRE) at its 5 '-end. This IRE allows iron regulatory protein 1A (IRP1A), the Drosophila ortholog of human iron regulatory protein 1 (IRP1), to bind to the Fer1HCH-RA mRNA [72-76]. Specifically, we used the IRP1A ${ }^{\text {C450S }}$ form [74], which is constitutively RNA-binding. We then designed a series of crRNAs that direct dCasFX to its target, Fer1HCH-RA, and tested whether immunoprecipitation of dCasFX would also pull down IRP1A. We transfected and lysed cells containing the dCasFX and crRNA components and mixed this lysate with a second sample obtained by lysing cells containing transfected Fer1HCH-RA mRNA and IRP1A ${ }^{\mathrm{C} 450 \mathrm{~S}}$. By combining the two lysates, the dCasFX/crRNA enzyme should bind to the Fer1HCH-RA mRNA/IRP1A ${ }^{\text {C450S }}$ complex. If the interaction occurs, immunoprecipitation of dCasFX (via its added HA tag) is expected also to pull down IRP1A ${ }^{\text {C450S }}$ (Fig. 4d). 
A key question for this strategy was how far the recognition site for dCasFX/crRNA needed to be away from the IRE to allow binding of both proteins, dCas13 and IRP1A, to the Fer1HCH-RA mRNA. To this end, we generated nine different crRNAs, representing binding sites spaced $\sim 150$ bases apart to roughly cover the entire $1.7 \mathrm{~kb}$ Fer1HCH-RA mRNA. One of the sites (crRNA \#3) partially overlapped with the IRE site, which served as a control to disrupt IRP1A binding. Using this strategy, we found that immunoprecipitation of dCasFX successfully pulled down IRP1A, as long as the cRNA binding site was sufficiently removed from the IRE. As expected, this interaction appeared to be dependent on the distance between the crRNA target site and IRE sequence, since an insufficient distance should cause steric hindrance between the two proteins (Fig. 4e). As a control, we used a non-targeting (NT) crRNA to ensure the interactions we observed were specific. The control showed that immunoprecipitation of dCasFX with a non-Fer1HCH-RA mRNA-targeting cRNA was not able to pull down IRP1A.

We also tested whether we can simply detect immunoprecipitated Fer $1 H C H-R A$ mRNA via real-time PCR (qPCR). In the absence of IRP1A, dCasFX appears to bind to the Fer $1 H C H-R A$ mRNA efficiently, and we found no significant differences between the nine different crRNAs (Additional file 1: Fig. S5A). Interestingly, when we repeated the experiment in the presence of IRP1A, we noticed a $\sim 4$-fold reduction of immunoprecipitated Fer1HCH-RA mRNA when we used cRNAs \#1-4 (Additional file 1: Fig. S5B). This is consistent with the results for co-immunoprecipitated IRP1A (Fig. 4e), suggesting that competition between IRP1A and dCasFX (bound to crRNAs \#1-4) affected the RNA-binding ability of both proteins. We conclude that dCasFX is a reliable tool to validate interactions between a protein and its candidate target RNA. In addition to RNA immunoprecipitation, dCasFX could potentially also used for other in vivo studies, such as locating a transcript of interest to elucidate its subcellular localization or for co-localization studies, or to determine whether a given protein is bound to its target RNA or unbound.

\section{Targeting mitochondrial RNAs via Cas13}

Like CRISPR/Cas9, Cas13 needs to form a complex with a crRNA before it can identify and cleave its target transcript $[22,23]$. Since the Cas13/crRNA complex harbors a single protein, it can be easily tagged with a mitochondrial targeting sequence to cleave RNA in mitochondria, which is not feasible with RNAi. Drosophila mitochondria contain multiple copies of circular DNA (mtDNA), which encode tRNAs, rRNAs, and polypeptides important for oxidative phosphorylation. The study of mitochondrial genes is important, because mutations in mtDNA can cause devastating human disorders, such as Leber's hereditary optic neuropathy, which causes blindness [77-79]. To modify CRISPR/Cas13 applications for mitochondrial-encoded transcripts, we added a sequence encoding an $\mathrm{N}$-terminal mitochondrial targeting peptide derived from the nuclear-encoded translocase of the inner mitochondrial membrane 23 (tim23) gene. For this approach, we generated a modified version of our highly efficient CasFX variant, which we termed CasFX ${ }^{\mathrm{mt}}$. The CasFX ${ }^{\mathrm{mt}} / \mathrm{crRNA}$ complex is predicted to be imported into the mitochondrial matrix, where it should bind to and cleave the target transcripts (Fig. 4f, g). 
To test the functionality and efficiency of the CasFX ${ }^{\mathrm{mt}}$ variant, we co-transfected CasFX $^{\mathrm{mt}}$ with constructs encoding a crRNAs against either mitochondrial cytochrome $c$ oxidase subunit I (mt:CoI, aka COXI) or mitochondrial cytochrome c oxidase subunit II (mt:CoII, aka COXII). Both COXI and COXII are highly expressed mitochondrialencoded genes critical for oxidative phosphorylation [80-82]. We analyzed the expression levels of COXI and COXII via qPCR as well as Western blots. To put these results into context, we generated RNAi samples against each of these targets and used the original CasFX ( $\mathrm{CasFX} \mathrm{X}^{\mathrm{O}}, \mathrm{O}=$ original) variant, which lacks the mitochondrial sequence, as a control. In our hands, RNAi targeting either COXI or COXII had no significant effect on the expression of these two transcripts. Similarly, CasFX $/$ crRNA produced no significant effects (Fig. 4h, i). In stark contrast, $\mathrm{CasFX}^{\mathrm{mt}}$ caused a 4-5-fold reduction of the COX transcripts and resulted in a substantial drop in protein levels as well (Fig. 4h, i). To ensure that this result was reproducible, we tested additional RNAi as well as crRNA sequences, all of which target COXI or COXII transcripts (Additional file 1: Fig. S2). In all cases, the observed results were comparable (Additional file 1: Fig. S5C-E), suggesting that $\mathrm{CasFX}^{\mathrm{mt}}$ is a useful tool to target mitochondrial-encoded transcripts.

\section{Cas13-ADAR2 for RNA modification}

One intriguing aspect of CRISPR/Cas13 has focused on the modification of RNA, which led to two approaches, namely " $\underline{R}$ NA editing for programmable $A$ to $I$ replacement" (REPAIR) and "RNA editing for specific $C$ to $U$ exchange" (RESCUE) [47, 50]. These methods allow for programmable adenosine-to-inosine editing as well as cytosine-to-uridine editing, respectively. The ability to modify genetic information at the RNA level may be advantageous because, unlike Cas9, which causes a permanent change in the genome, RNA modifications via Cas13 are reversible due to RNA turnover $[8,12,39,74]$. As such, Cas13-based approaches may be suitable for future therapies, where Cas13 could be used to repair missense mutations in transcripts without affecting a patient's genome.

In the REPAIR systems used in mammalian cells, the nuclease-dead PspCas13b was fused to the RNA-modifying domain of Adenosine Deaminase Acting on RNA 2 (ADAR2). In their original approach, Cox et al. found that the first REPAIR version (REPAIRv1) had substantial off-target activity. Subsequently, they generated REPAIRv2, which harbored two point mutations in the ADAR2 domain (T375G and E488Q). This version showed high specificity and robustness in mammalian cells [50].

Given its success in mammalian cell systems, we wondered whether a Cas13-ADAR fusion would be functional in Drosophila. The insect ADAR protein appears to function similarly to its human counterpart [83], suggesting that constructs based on mammalian ADAR2 would work in Drosophila. We first fused the above-described dCasFX to the mutant human ADAR2 domain that carries equivalent mutations as the REPAIRv2 we mentioned earlier. We refer to this construct as FREPAIRv2 $(\mathrm{F}=$ fruit fly) and tested for its editing efficiency (Fig. 5a). To test for Cas13-ADAR2 activity, we generated a system that uses a dual-reporter transgene in the Drosophila embryo cell line Sg4-PP-27F. Similar to the earlier described Sg4_CD line; this cell line carries the independently expressed eCFP and DsRed transcription units in the genome, each with their own actin5 promoters. However, unlike the Sg4_CD line, we introduced a point mutation 


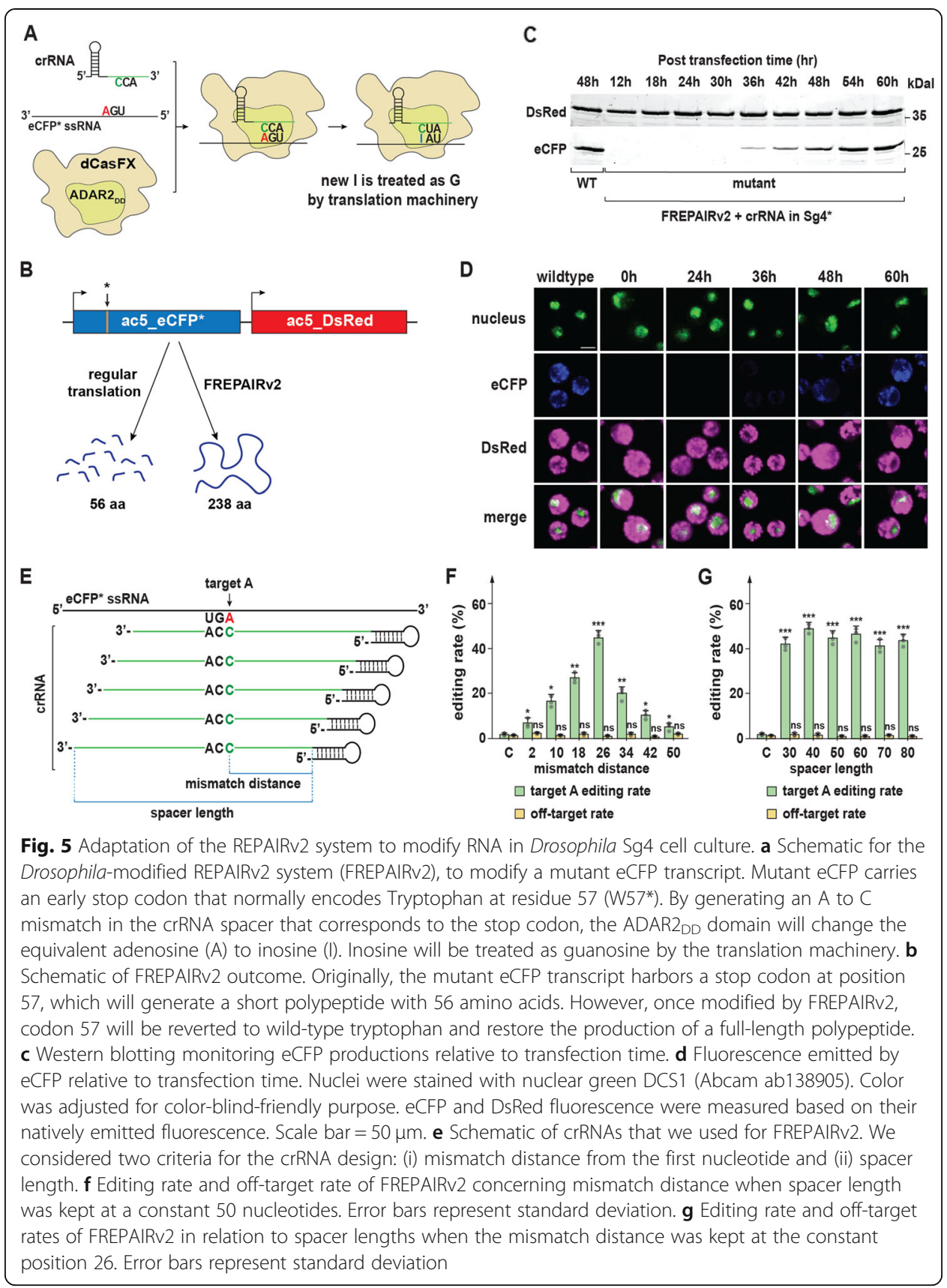

into the eCFP coding region that converts a tryptophan residue 57 (W57*) TGG into an early stop codon (TGA), which we refer to as eCFP*. Also, we termed this new cell line "Sg4"” line to distinguish it from the original Sg4_CD (Additional file 1: Fig. S1B). Next, we co-expressed FREPAIRv2 and an eCFP-crRNA, which carries a single mismatch $\mathrm{A}$ to $\mathrm{C}$ at the position that corresponds to the introduced stop codon (Fig. $5 \mathrm{a}$, b). If the FREPAIRv2 is capable of editing its target RNA encoded by $e C F P^{*}$, the stop codon should be reverted to the wild-type tryptophan residue (W57), and the resulting full-length eCFP should be detectable via Western blotting and, if efficiency is sufficiently high, via fluorescence from the restored CFP. Using this strategy, we found that we were able to detect fluorescence at a wavelength of $405 \mathrm{~nm}$ as early as $36 \mathrm{~h}$ after 
transfection, indicating that detectable levels of eCFP had been produced. eCFP fluorescence continued to increase, with substantially higher levels at the 60-h time point (Fig. 5d). When we conducted Western blots to validate these data, we saw corresponding results, with detectable eCFP protein at $36 \mathrm{~h}$ and progressively higher levels from 42 to $60 \mathrm{~h}$ after transfection (Fig. 5c). We conclude that Cas13-ADAR2 works effectively in Drosophila and can be used to modify target mRNAs, such as reverting transcripts carrying missense mutations without altering the genome.

For the above approach, we followed a similar path that was used in the original study [50] where the mismatch $(\mathrm{C} \rightarrow \mathrm{A})$ was placed in the center of the crRNA spacer, measured at the 26th nucleotide of 50 nucleotides (nt) spacer, relative to the stem loop-forming direct repeat of the crRNA. To evaluate the editing efficiency in correlation to mismatch position and spacer length, we tested a series of crRNA constructs with the same spacer length of $50 \mathrm{nt}$; however, we changed the relative mismatch distance to the hairpin by increments of $8 \mathrm{nt}$ (Fig. 5e). We then performed reverse transcription and sequenced a minimum of ten randomly selected eCFP cDNAs per construct. This was followed by sequencing to assess the fraction of clones that harbored the repaired codon for tryptophan \#57, expressed as editing rate (Fig. 5f). Based on our findings, the crRNA that carried the mismatch at position 26 relative to the hairpin ("mismatch distance", Fig. 5e) resulted in the highest efficiency (Fig. 5f), consistent with other studies [50]. We then tested the effect of varying spacer length while keeping the mismatch distance at $26 \mathrm{nt}$. We tested spacer lengths from 30 to $80 \mathrm{nt}$, and in all cases, we observed similar efficiencies, all of which were comparable to a 50-nt spacer (Fig. 5g). Based on these findings, we conclude that FREPAIRv2 works best when using a mismatch distance of $26 \mathrm{nt}$, whereas the spacer length did not appear to affect the editing efficiency [50].

To evaluate the off-target tendencies of FREPAIRv2 in Drosophila cells, we examined the cDNA sequences for additional $\mathrm{A} \rightarrow \mathrm{I}$ modifications, which is straightforward since inosine is recognized as guanosine by the reverse transcriptase. However, we scored any unpredicted sequence deviations as potential off-target events and plotted them relative to the mismatch distances and spacer lengths (Fig. 5f, g). This strategy revealed that some off-target effects persisted, albeit at a low level across all crRNAs that we tested. Given that these effects are random, and distributed across multiple RNA molecules, it appears likely that this off-target activity has no or inconsequential impact on phenotypes. However, future studies are needed to improve the specificity of this editing system further.

\section{Generation and characterization of transgenic Cas13 flies}

Our data demonstrated that Cas13 works well in Drosophila Sg4 cells and can be used for purposes beyond RNA cleavage. We next sought to generate transgenic fly lines carrying Cas13 variants and characterize their efficacy in vivo. We were also interested in creating a system that allows for temporal and spatial control over Cas13 expression. To this end, we have previously created a Drosophila toolkit for CRISPR/Cas9 based on Gateway-compatible cassettes that allow researchers to insert specific enhancers that drive the expression of the Cas transgene in a tissue of interest $[8,59]$. While this generates more upfront work compared to Gal4/UAS-based systems driving the expression 
of Cas9 [12, 37], it does simplify the downstream workflow. Also, it reduces unspecific effects since one requires fewer transgenes to build the necessary fly genotype. We, therefore, decided to create a similar Cas13 toolkit. In total, we manufactured two general Cas13 vectors, one based on CasFB and one that uses CasFX, both of which displayed the highest catalytic efficiency in Sg4_CD cells. For our in vivo strategy, we limited our efforts to constructs that would interfere with RNA expression (Additional file 1: Fig. S6A). Based on these all-purpose vectors, we then generated four transgenic lines for further characterization, named here act_CasFB, UAS-CasFB, act_CasFX, and UAS-CasFX (Additional file 1: Fig. S6A). For the generation of crRNAs, we used the previously described multiplexed pCFD5 vector and implemented changes suitable for Cas13 crRNA processing [12]. We refer to the new plasmids as i) pC13B, which expresses CasFB-compatible crRNAs under control of the U6:3 promoter and ii) pC13X, which expresses CasFX-compatible crRNAs under control of the U6:3 promoter (Additional file 1: Fig. S6B, C). Both plasmids will ubiquitously express the tRNA:crRNA units. As the tRNA is processed, its cleavage will result in the release of mature crRNAs that form complexes with Cas13 enzymes. The cloning procedures for these new crRNA plasmids are overall similar to those for the pCFD5 vector, but since some differences exist, we include a detailed protocol in the supplementary material (see supplemental method S1).

To evaluate the efficiency of our transgenic Cas13 constructs in vivo, we generated seven transgenic crRNAs targeting three genes that we study in our lab. This includes phantom ( $\mathrm{phm}$ ) and disembodied (dib), two well-characterized genes involved in ecdysone synthesis in Drosophila [84, 85] as well as the third gene, Iron Regulatory Protein $1 A$ (IRP1A), a gene critical for cellular iron homeostasis [74, 86]. Classic mutants of phm and dib display embryonic lethality while IRP1A mutant animals die as first instar larvae (L1) [8, 59, 74, 84, 87]. In contrast, using PG-specific somatic CRISPR/Cas9 strategies, $p h m^{g R}$ (gRNA for CRISPR Cas9) caused L1 arrest, while $d i b^{g R}$ and $I R P 1 A^{g R}$ both caused third instar (L3) larval arrest (Fig. 6a-c) [8, 59, 74]. In addition, PGspecific disruption of IRP1A via somatic CRISPR/Cas9 caused a porphyria-like phenotype due to iron deficiency (Fig. 6d) [74].

When we crossed the Cas13-compatible crRNAs (referred to as 13B for CasFBcompatible cRNAs and 13X for CasFX-compatible crRNAs) targeting either phm, dib, or IRP1A with either ubiquitously expressed or PG-specific Cas13 variants, we observed the same developmental defects we found with our previous strategies (Figs. 6a-d, S2, and S7, Additional file 5: Table S4), indicating that Cas13 worked effectively in Drosophila. The fact that $\mathrm{phm}^{13 B}, \mathrm{phm}^{13 X}, \mathrm{dib}^{13 B}$, and $\mathrm{dib}^{13 X}$ individuals were rescued to adulthood when reared on 20E-supplemented media $[8,59]$ and that $I R P 1 A^{13 B}$, as well as $I R P 1 A^{13 X}$ animals, reached adulthood when dietary iron was provided [74], strongly suggested that the activity Cas13 was highly specific (Figs. 6a-c and S7).

In addition to the above phenotypic analysis, we evaluated dib expression levels via qPCR. We compared the results to other tissue-specific loss-of-function techniques, including samples from two independent RNAi lines and samples from one line where we used transcriptional interference via dead Cas9 (dCas9) to target dib. We found that the two RNAi lines reduced dib expression by 30-40\%, whereas the CRISPRi approach via dCas9 lowered dib expression by 50-60\%. Concerning the new Cas13 lines, CasFB reduced dib expression by 55-65\%, equivalent to the dCas9 data. Remarkably, CasFX 


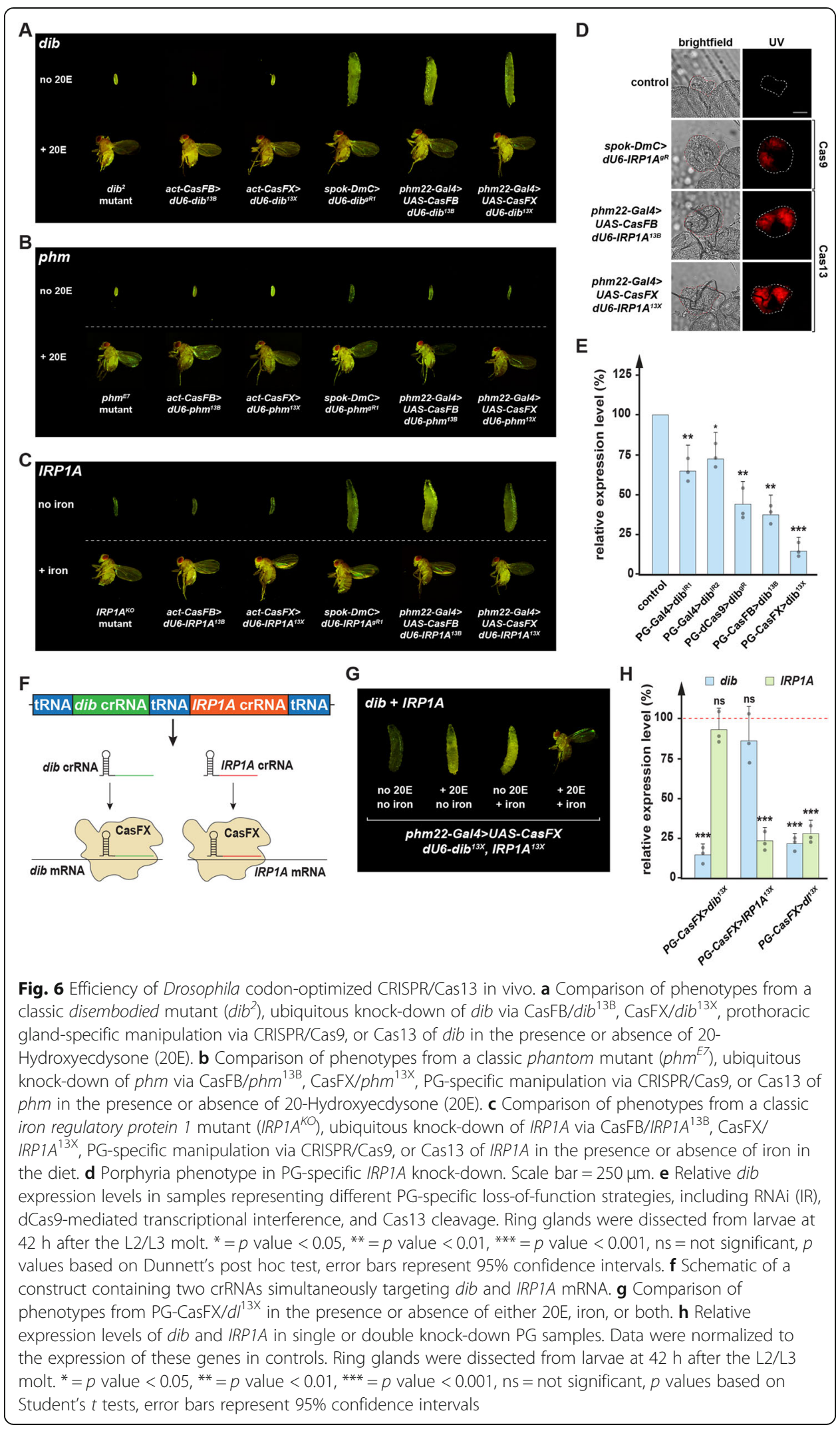


showed the strongest knock-down and robustly reduced dib expression by $80-90 \%$ (Fig. 6e). These data indicated that Cas13 transgenes work in vivo and may exceed the efficacy of other techniques.

We also tested the ability to target multiple transcripts with a single transgene. For this, we used the $\mathrm{pC} 13 \mathrm{X}$ vector and generated a dual-crRNA transgenic line (termed $\mathrm{dI}^{13 \mathrm{X}}$ ) that ubiquitously expressed a crRNA-targeting dib mRNA as well as a crRNA targeting the IRP1A transcript (Fig. 6f). Target sites for either of these transcripts were the same as before (Figs. 6a, c and S2). As expected, the animals arrested development at the L3 stage, similar to targeting the $d i b$ and IRP1A transcripts individually. Consistent with this, neither 20E- nor iron-supplementation alone could rescue these double knock-downs; however, a diet supplemented with both 20E and iron caused a significant rescue to adulthood (Fig. 6g). This makes sense since the two cRNAs interfered with ecdysone production and the regulation of cellular iron homeostasis. To assess whether the simultaneous knock-down of two genes was as efficient as targeting these genes individually, we evaluated $d i b$ and IRP1A expression levels via qPCR. We found no significant difference in any of these approaches suggesting that there is no penalty when targeting two genes at the same time (Fig. 6h).

\section{Discussion}

\section{RNA degradation efficiency of Cas13 in Drosophila}

We evaluated eleven variants of each reported Cas13 ortholog in Drosophila Sg4 cells, including the well-characterized variant from the original studies and ten Drosophilaoptimized variants. Among all Cas13 enzymes tested, we observed a wide range of efficiencies, even between the versions from the same ortholog. Among them, CasRX and its Drosophila-optimized variants CasFX appeared to have the highest efficiency. For the Cas13a and Cas13b variants, we also identified the optimized variants with reliable efficiency. Even though they were less efficient than CasFX, these variants may still prove useful in circumstances where only a moderate knock-down is desired. On the other hand, Cas13c variants did not significantly alter the expression of target transcripts. We hypothesize that this was caused by several factors: (i) Cas13c is the least characterized Cas13 enzyme, and it might use a mechanism that differs from the other Cas13 enzymes. (ii) Even though the low efficiency of Cas13c was in agreement with previous studies conducted in other species, we cannot rule out the possibility that the Cas13c variants we used were not ideally suited for Drosophila, and (iii) Cas13c might still require a PFS for optimal activity in the fruit fly. Future studies will need to address this.

We noticed that the expression of the PspCas13b and CasRX variants resulted in considerable toxicity when animals were homozygous for these transgenes, causing lethality during the first (L1) or second (L2) instar larvae (Additional file 1: Fig. S8). Interestingly, animals heterozygous for PspCas13b and CasRX transgenes showed no significant lethality. In contrast, animals homozygous for our Drosophila-optimized Cas13 transgenes, namely CasFB and CasFX, showed only moderate lethality, with 51 to $58 \%$ reaching adulthood, respectively ( $80-85 \%$ is expected in wild-type populations). As expected, animals heterozygous for these transgenes appeared normal (Additional file 1: Fig. S8). The lethality of Cas13 transgenic animals was also reported in a recent 
study [88], similar to the results of early versions of Cas9 in Drosophila [8, 10]. Since we observed a wide range of efficiencies between the variants, it is possible that each variant also exhibits different levels of toxicity. While the reasons for the relatively high lethality of the original PspCas13b and CasRX constructs (in a homozygous setting) remain unclear, our data suggest that each variant is unique and that perhaps using codon-optimized versions help to reduce the toxicity associated with Cas13.

\section{Beyond RNA cleavage}

A few studies have shown that Cas13 may be useful in a broad range of applications and not just RNA cleavage. In this study, we have demonstrated that dCasFX can validate RNA-protein interactions by using an appropriately designed crRNA. We also showed that by adding a mitochondrial localization sequence, one could recruit the $\mathrm{CasFX}^{\mathrm{mt}} / \mathrm{crRNA}$ complex into mitochondria and target mitochondrial-encoded transcripts. We also adopted the REPAIRv2 system from mammalian cell culture into Drosophila Sg4 cells and showed that this system, FREPAIRv2, can efficiently modify target transcripts with an overall low off-target rate. We have not tested other potential applications; however, in theory, Cas13 can be modified for many approaches to study RNA, including splicing, transcript stabilization, or RNA localization.

Cas13 may have far-reaching implications for simplifying diagnostics. Recently, the outbreak COVID-19 caused by SARS-CoV-2 has resulted in a global health threat. To develop a fast test for COVID-19, the specific high-sensitivity enzymatic reporter unlocking (SHERLOCK) protocol, a recently developed Cas13-based diagnostic test for infectious diseases, can detect the virus in $50 \mathrm{~min}[89,90]$ (https://mcgovern.mit.edu/2 020/02/14/enabling-coronavirus-detection-using-crispr-cas13-an-open-access-sherlockresearch-protocol/). In an independent study, CRISPR/Cas13 was also used to detect SARS-CoV-2 [91]. Together, these studies demonstrate the enormous potential of Cas13 as a diagnostic and therapeutic tool.

\section{From in vitro to in vivo}

A significant part of the work presented here was based on cell culture experiments. These approaches were ideal to economically evaluate the efficiencies of multiple Cas13 versions in Drosophila. However, our ultimate goal is to establish CRISPR/Cas13 approaches for in vivo studies in model organisms, which has not been accomplished yet. Based on our results of transgenic CRISPR/Cas13 flies, CasFX and CasFB can efficiently target and cleave transcripts of interest in vivo, and as such, represent a compelling alternative to existing methods. This study may also help scientists working with other model organisms to optimize their approach for implementing Cas13 in vivo.

\section{The CRISPR/Cas13-based toolkit}

This study has generated two collections of Cas13/crRNA toolkits to study in either cell culture or organisms. For the cell culture toolkit, we have produced the pC13cr01 vectors, which allow the co-transfection of Cas13 variants and the crRNA corresponding to the target transcript. With this vector, one only needs to digest the crRNA backbone with the BbsI enzyme and clone the target site for the crRNA, similar to the generation of the Cas9-compatible gRNA system in pCFD5 or pCFD6 plasmids. For in vivo work, 
we also established a similar system with Cas13 transgenes already available from our study. Researchers will need to generate their crRNAs against the target transcript. For this, we provide the $\mathrm{pC} 13 \mathrm{~B}$ and $\mathrm{pC} 13 \mathrm{D}$ vectors with the same cloning procedure as pCFD5. We also provided a supplemental method section with a detailed description of the cloning procedures. On the other hand, the UAS-based versions of Cas13 transgenes will also allow scientists to spatially and temporally manipulate Cas13 activity and study transcript of interest at desired tissues.

\section{Conclusions}

Just like CRISPR/Cas9 allows for the manipulation of DNA, Cas13 enables us to target any transcript of interest. This is beneficial for approaches where researchers do not want to alter the DNA of the gene of interest, since Cas13 controls gene expression on the RNA level, similar to RNAi. Furthermore, current evidence suggests that Cas13, especially variants from the Cas13d family, display minimal off-target tendencies, and this might help quell concerns regarding RNA targeting. Even though it might be too early to make conclusions about the off-target activity of Cas13, we believe that its high specificity holds excellent promise for future applications. Also, the ability to modify Cas13, such as targeting Cas13 to mitochondria, further expands the range of future applications for this methodology.

\section{Methods}

\section{Generation of Drosophila-optimized Cas13 orthologs (DmCas13)}

To generate fruit fly codon-optimized Cas13 variants, the original Cas13 nucleotide sequences were evaluated by using two independent web tools: (i) ATGme (https:// atgme.org) and (ii) OPTIMIZER (http://genomes.urv.es/OPTIMIZER) [92, 93] with the customized codon usage frequency specific for Drosophila [94-96]. The two indices, namely the codon adaption index (CAI) and the effective number of codons (ENC), were used to obtain the optimized sequences. CAI has value ranges from 0 to 1 and is used to evaluate the similarity between codon usage of a gene and codon usage of the reference group [97]. Thus, at least in theory, the higher the CAI value, the higher is the gene expression $[98,99]$. On the other hand, ENC is a measure of codon usage bias with values between 20 and 61 . Since the expression of a gene is usually dependent on the availability of tRNA species, one would expect that genes with higher expression will use a smaller subset of codons recognized by the most abundant tRNAs, resulting in lower ENC values [100]. Taking these two factors into consideration, we picked the top 10 variants per Cas13 subtype for further investigation (Additional file 2: Table S1). We reasoned that it would not suffice just to choose the top-scoring variant, and therefore, we also selected other high-scoring sequences. We generated the selected variants via a combination of mutagenesis of the original Cas13 sequences and fusing gBlocks gene fragments from Integrated DNA Technologies (IDT) (Additional file 4: Table S3).

\section{Design and generation of target crRNAs}

The very first Cas13 proteins that were characterized in bacteria required a sequence constraint, the PFS, to ensure target cleavage efficiency. This includes Leptotrichia shahii Cas13a (LshCas13a), Bergeyella zoohelcum Cas13b (BzoCas13b), and Prevotella 
buccae Cas13b (PspCas13b) [50, 53]. However, further investigation of PspCas13b in mammalian and plant and other Cas13 orthologs showed high target RNA degradation efficiencies even in the absence of PFS [23, 46, 49, 52]. While this gives researchers some flexibility over target site selection, it is necessary to consider the secondary structure of target transcripts, since this negatively affected knock-down efficiency [23, 50]. To assess secondary structures, we used two independent online tools, namely RNAfold (http://rna.tbi.univie.ac.at/cgi-bin/RNAWebSuite/RNAfold.cgi) and RNA structure (https://rna.urmc.rochester.edu/RNAstructureWeb/Servers/Predict1/Predict1. html) [101-104]. Besides, we also used the siRNA design tool RNAxs (http://rna.tbi. univie.ac.at/cgi-bin/RNAxs/RNAxs.cgi) to find the regions of transcripts with good accessibility to narrow down the target region space for designing gRNAs [105]. For the case of Cas13a orthologs, we compared the target sequences with the online CRISPRRT tool (http://bioinfolab.miamioh.edu/CRISPR-RT/interface/C2c2.php) [106]. The crRNA cassette was amplified and cloned into a pre-digested expression vector backbone via the Gibson reaction. All crRNAs used in this study were driven by the Drosophila U6:3 promoter (dU6:3). For more information regarding crRNA cloning, see supplement method S1.

\section{Generation of transfection plasmids}

For a list of plasmids, we generated for this study, see Additional file 3: Table S2. The original plasmids we used for this project were obtained from Addgene: pCFD3 (\#49410), pCFD5 (\#73914), pACG:eCFP (\#32597), pDsRed-attP (\#51019), Ac5-Stable2Neo (\#32426), pC0056-LwaCas13a-msfGFP-NES (\#105815), pC0040-LwaCas13a crRNA backbone (\#103851), pC0046-EF1a-PspCas13b-NES-HIV (\#103862), pC0043PspCas13b crRNA backbone (\#103854), pC0054-CMV-dPspCas13b-longlinkerADAR2DD (E488Q/T375G) (103870), pXR001: EF1-CasRX-2A-eGFP (\#109049), pXR004: CasRX pre-gRNA cloning backbone (\#109054), pBID-UASc (\#35200), [10, 12, $23,35,46,50,107-110]$. We also obtained plasmids from the Drosophila Genetic Resource Center (DGRC): pAFW (\#1111), pAHW (\#1095), act-PhiC31-integrase (\#1368). We also used plasmids we previously generated, enDmC, to generate some constructs for this study [8]. pMT-Gal4-puro plasmid was a kind gift from Christoph Metzendorf (University of Uppsala). All fragments used for the cloning step were amplified via PCR using Q5 high-fidelity DNA polymerase (NEB \#M0491S) (Additional file 4: Table S3) and fused together via Gibson assembly reaction [111].

\section{Generation of transgenic cell lines}

The original Sg4-PP-27F (\#238) cell culture line was obtained from Drosophila Genetics Resource Center (DGRC) and grown in the HyClone SFM4 Insect cell culture (SFM4) medium (GE Lifesciences SH30913.02) with 1\% (v/v) streptomycin-penicillin (Sigma P4333) following standard procedures (Invitrogen). To generate the transgenic Sg4_CD cell line, Sg4-PP-27F cells were co-transfected with two different plasmids, where one plasmid contained the PhiC31 integrase gene, and the other was the dualreporter plasmid (Additional file 1: Fig. S1A). The dual-reporter transgenic construct also contained a $N e o^{R}$ gene, which allows for resistance to Geneticin G418 (Sigma 4727878001). Then, $48-72 \mathrm{~h}$ after co-transfection, cells were washed and grown in 
SFM4 medium supplemented with G418 at the final concentration of $200 \mu \mathrm{g} / \mathrm{ml}$. Transfected cells were maintained on this type of medium (SFM4 with $1 \%$ streptomycinpenicillin and $200 \mu \mathrm{g} / \mathrm{ml} \mathrm{G418)} \mathrm{for} \mathrm{at} \mathrm{least} \mathrm{four} \mathrm{passage} \mathrm{rounds} \mathrm{before} \mathrm{being} \mathrm{tested} \mathrm{for}$ the integration of transgenic constructs via Sanger sequencing.

\section{DNA extraction from cells}

Cells were grown, and DNA was extracted as previously described [59]. In brief, cells were collected as pellets and filled with $20 \mu \mathrm{l}$ of DNA extraction buffer $(10 \mathrm{mM}$ Tris$\mathrm{HCl} \mathrm{pH}$ 8.2, $25 \mathrm{mM} \mathrm{NaCl}, 1 \mathrm{mM}$ EDTA $\mathrm{pH}$ 8.0, 0.2\% v/v Triton X-100, $1 \times$ proteinase $\mathrm{K}$ (AM2546)). The mixture was vortex for $3 \times 30 \mathrm{~s}$ and incubated at $37^{\circ} \mathrm{C}$ for $30 \mathrm{~min}$ before heat-inactivated at $95^{\circ} \mathrm{C}$ for $5 \mathrm{~min}$. Cell lysates were centrifuged at $12,000 \times g$ at $4{ }^{\circ} \mathrm{C}$ for $5 \mathrm{~min}$, and the supernatant was transferred to a new collection tube. One microliter of supernatant was used for PCR amplification at the genomic region spanning target sites. PCR products were purified using the HighPrep ${ }^{\mathrm{ma}}$ PCR reagent from MagBio (AC-60005) following the manufacturer's protocol.

\section{Cell culture transfection}

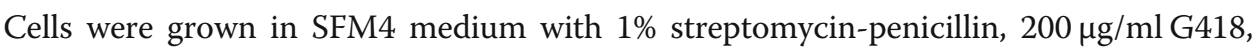
and transfected by the calcium phosphate-based method (Invitrogen). To study the effects of different Cas13 variants, puromycin was added to the medium on the second day after transfection at the final concentration of $5 \mu \mathrm{g} / \mathrm{ml}$, similar to what was previously used $[112,113]$. Cells were collected 7 days after transfection to ensure the turnover of already translated eCFP polypeptides [114]. Transfected cells were washed in ice-cold $1 \times$ PBS for $3 \times 5$ min and collected for later experiments.

\section{Cell immunostaining}

On the first day of the transfection experiment, coverslips were pre-cleaned in $70 \%$ ethanol and placed into a transfection plate (Sigma CLS3516). Cells were then seeded and transfected following the standard procedures (Invitrogen). This allows cells to adhere to the coverslips for subsequent immunostaining. Subsequent transfection procedures were carried out as described in the cell culture transfection section. Seven days after transfection, coverslips were transferred to a clean transfection plate for immunostaining, while cells in the supernatants were collected for cell lysis and protein extraction.

Samples were fixed in 1× PBS 4\% formaldehyde (Thermo Fisher \#28906) for $15 \mathrm{~min}$ at room temperature (RT) with gentle shaking followed by washing in $1 \times$ PBS $0.3 \%$ Triton (Sigma \#T9284) (PBS3T) for $3 \times 10 \mathrm{~min}$. Samples were blocked at RT for $30 \mathrm{~min}$ in blocking solution (1× PBS3T 5\% normal goat serum (Abcam ab138478)) and incubated in primary antibody dilution buffer (antibody diluted in $1 \times$ PBS3T and 1\% BSA) for $1 \mathrm{~h}$ at RT. Samples were then washed in $1 \times$ PBS3T for three times with 10 min each, incubated in secondary antibody dilution buffer for $1 \mathrm{~h}$ at RT, and then washed in $1 \times$ PBS3T with either 1:50,000 DAPI (Cell Signaling \#4083) or 1:2000 Nuclear Green DCS1 (Abcam ab138905) for $3 \times 10$ min. Samples were mounted in Vectashield mounting medium (\#VECTH1000). Pictures were taken on Nikon Eclipse 80i Confocal C2+ microscope/camera. We used the following reagents: a monoclonal mouse anti-HA-tag 
antibody (Abcam ab18181) at the ratio of 1:1000 for 3xHA tagged Cas13 orthologs, mitotracker green (Cell signaling 9074S) at the concentration of $400 \mathrm{nM}$ for staining mitochondria, monoclonal mouse anti-MTCO1 (Abcam ab14705) at the ratio of 1 : 2000, and monoclonal rabbit anti-MTCO2 (Abcam ab79793). Secondary antibodies were obtained from Abcam and used at the ratio of 1:2000, including goat anti-mouse IgG H\&L Alexa Fluor 488 (ab150113), goat anti-mouse IgG H\&L Alexa Fluor 555 (ab150114), and goat anti-rabbit IgG H\&L Alexa Fluor 555 (ab150078). eCFP and DsRed signals were captured based on their fluorescence properties without antibody staining. For quantification of the eCFP signal, the mean pixel values of the images were analyzed using ImageJ as the corrected total cell fluorescence (CTCF) following the formula: CTCF $=$ selected cell intensity $-($ area of the chosen cell $\times$ background intensity). The CTCF values were averaged from all biological replicates and normalized to the normalized average CTCF values of no-targeting (NT) crRNA samples.

\section{Western blotting of cell extracts}

For cell lysis and Western blotting, 7-day post-transfection cells were collected by centrifugation at $1000 \times g$ for $10 \mathrm{~min}$ at $4{ }^{\circ} \mathrm{C}$ and supernatant was removed as much as possible. Cells were washed in ice-cold $1 \times$ PBS for $3 \times 10 \mathrm{~min}$ and lysed in $90 \mu \mathrm{l}$ lysis buffer ( $1 \times$ PBS, $1 \%$ Triton, $1 \times$ proteinase $\mathrm{K}$ inhibitor) by vortexing for $15 \mathrm{~s}$ every $10 \mathrm{~min}$ for up to $1 \mathrm{~h}$. Cell lysate was mixed with fresh $4 \times$ Laemmli buffer $(0.25 \mathrm{M}$ Tris $\mathrm{pH} 6.8,8 \%$ SDS, $40 \%$ glycerol, $25 \% \beta$-mercaptoethanol, $0.2 \%$ bromophenol blue) at the ratio of 3:1 $(\mathrm{v} / \mathrm{v})$. Forty microliters of the mixture (1/3 total volume) was loaded on $12.5 \%$ SDS gel. Later steps, including gel electrophoresis and Western blotting, were carried out following the manufacturer's (Abcam) instructions. To detect eCFP, monoclonal rabbit antiGFP-tag antibodies (Invitrogen G10362) were used at a ratio of 1:1000, followed by incubation with a goat anti-rabbit IgG H\&L HRP secondary antibody (Abcam ab97051) at a ratio of 1:20,000. To detect DsRed, monoclonal mouse anti-DsRed antibody (Santa Cruz sc-390909) was detected at the ratio of 1:1000, followed by incubation with a goat anti-mouse IgG H\&L HRP secondary antibody (Abcam ab97023) at the ratio of 1:20, 000. To detect COXI and COXII, monoclonal mouse anti-MTCO1 antibody (Abcam ab14705) and monoclonal rabbit anti-MTCO2 antibody (Abcam ab79393), respectively, were used at a ratio of 1:500. We used monoclonal mouse anti- $\beta$-tubulin antibodies (Sigma 05-661) at a ratio of 1:10,000 to detect tubulin, which served as a loading control. Blots were scanned for image acquisition with a ChemiDoc imaging system (BioRad), and band intensity was measured using ImageJ.

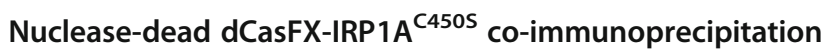

The dCasFX/crRNA complex and IRP1A ${ }^{\mathrm{C} 450 \mathrm{~S}} / \mathrm{Fer} 1 \mathrm{HCH}$ RA cDNA were transfected independently. In one sample, dCasFX and the crRNA corresponding to the Fer1HCHRA transcript were cloned into the same plasmid pC13cr01 (Additional file 1: Fig. S1D), while in another approach, IRP1A ${ }^{C 450 S}$ and Fer1HCH RA cDNA were similarly cloned into the same plasmid as pC13cr01. IRP1A ${ }^{\mathrm{C} 450 \mathrm{~S}} / \mathrm{Fer} 1 \mathrm{HCH}-\mathrm{RA}$ co-transfection was carried out at a 10x higher ratio compared to each dCasFX/crRNA transfection alone. Transfected samples were lysed using $200 \mu \mathrm{l}$ lysis buffer (1× PBS, 1\% Triton, $1 \times$ proteinase $\mathrm{K}$ inhibitor) by vortexing for $15 \mathrm{~s}$ every $10 \mathrm{~min}$ for up to $1 \mathrm{~h}$. Lysates of 
samples transfected with IRP1A ${ }^{\mathrm{C} 450 \mathrm{~S}} / \mathrm{Fer} 1 \mathrm{HCH}-\mathrm{RA}$ were combined and evenly distributed among ten groups of dCasFX/crRNA lysate. This ensured that each lysate had a similar amount of IRP1A ${ }^{\mathrm{C} 450 \mathrm{~S}} / \mathrm{Fer} 1 \mathrm{HCH}-\mathrm{RA}$ complex. The mixed lysate was incubated with pre-crosslinked HA Dynabeads protein G (Invitrogen 10004D) following the manufacturer's directions. Samples were eluted in $4 \times$ Laemmli buffer (0.25 M Tris pH 6.8, $8 \%$ SDS, $40 \%$ Glycerol, 25\% $\beta$-mercaptoethanol, $0.2 \%$ bromophenol blue).

\section{Drosophila stocks and husbandry}

We obtained the following stocks from the Bloomington Drosophila Stock Center: $w^{1118}$ (\#3605), dib ${ }^{2} / T M 3$ Sb $^{1}$ (\#2776), phm ${ }^{E 7} / F M 7 c$ (\#2208), $y^{1} v^{1}$ P[nos-PhiC31.NLS]X; attP4O(II) (\#25709), $y^{1} v^{1} P[n o s-P h i C 31 /$ int.NLS]X; attP2(III) (\#25710). Stocks UAS-dibRNAi (1) (\#101117), UAS-dib-RNAi (2) (\#16827), and UAS-phm-RNAi (\#108359) were obtained from the Vienna Drosophila Resource Center. $y^{2} c h o^{2} v^{1}$ (TBX-0004), $y^{2} c^{2} o^{2} v^{1}$; sco/CyO (TBX-0007), $y^{2}$ cho $^{2} v^{1} / Y^{h s-h i d} ; S p / C y O$ (TBX-0008), $y^{2} c h o^{2} v^{1}$; Sp hs-hid/CyO (TBX-0009), and $y^{2} c h o^{2} v^{1}$; $\operatorname{Pr} \mathrm{Dr} / \mathrm{TM} 6 \mathrm{C}, \mathrm{Sb} \mathrm{Tb}$ (TBX-0010) were obtained from the National Institute of Genetics of Japan (NIG). act_DmCas13B/CyO GFP, UASDmCas13B, act_DmCasRX/CyO GFP, UAS-DmCasRX, $y^{1} v^{1} ; P[p C F D 5$ dib.KO dgRNA]attP40 (dU6-dib ${ }^{\mathrm{gR} 13 \mathrm{~B}}$ ), $y^{1} v^{1} ; P\left[p C F D 5\right.$ dib.KO dgRNA]attP4O (dU6-dib ${ }^{\mathrm{gR} 13 \mathrm{D}}$ ), $y^{1} v^{1} ; P\left[p C F D 5 \quad\right.$ dib.KO dgRNA]attP4O (dU6-phm $\left.{ }^{\mathrm{gR} 13 \mathrm{~B}}\right), \quad y^{1} v^{1} ; P[p C F D 5$ dib.KO dgRNA]attP40 (dU6-phm $\left.{ }^{\text {gR13D }}\right), \quad y^{1} v^{1} ;$ P[pCFD5 dib.KO dgRNA]attP40 (dU6IRP1A ${ }^{\text {gR13B }}$ ), and $y^{1} v^{1} ; P\left[p C F D 5\right.$ dib.KO dgRNA]attP40 (dU6-IRP1A ${ }^{\text {gR13D }}$ ) were generated by our lab. spok_DmC/TM3,Ser.GFP (spok_DmC), $y^{1} v^{1} ; P[p C F D 5$ dib.KO dgRNA]attP4O (dU6-dib ${ }^{\mathrm{gR} 1}$ ), $y^{1} v^{1} ; P\left[p C F D 5\right.$ dib.KO dgRNAJattP4O (dib TSS ${ }^{-110}$ ), $y^{1} v^{1}$; P[pCFD5 dib.KO dgRNA]attP40 (dU6-phm ${ }^{\mathrm{gR} 1}$ ), P[pCFD5 dib.KO dgRNA]attP4O (dU6IRP1A $^{\mathrm{gR}}$ ), IRP1A ${ }^{K O} / T M 6 B, H u$, and $T b$ were previously generated by our lab [8, 74]. $y^{1} w^{*} P\left[\right.$ nos-PhiC31.NLS]X; attP4O(II) and $y^{1} w^{*} P[$ nos-PhiC31/int.NLS]X; attP2(III) were gifts from the BestGene Inc. phm22-Gal4 was a kind gift from Michael O'Connor's lab. Stocks were maintained on a cornmeal diet unless otherwise specified.

\section{Survival studies}

Experiments were carried out as previously described [8, 59, 74]. In brief, 50 embryos per replicate were collected in $1-\mathrm{h}$ intervals and transferred to vials containing appropriate media. Larval survival was scored for every stage. At least three independent crosses ( $=$ three biological replicates) were carried out per experimental condition. Modified media were prepared by adding compounds (e.g., iron or 20E) during the preparation process. For iron-enriched media, a $1 \mathrm{M}$ stock solution of ferric ammonium citrate (FAC) (Sigma \#F5879) was used to make a medium with a final concentration of $1 \mathrm{mM}$ FAC. For 20-hydroxyecdysone (20E)-supplemented media, the final concentration was $0.33 \mathrm{mg} / \mathrm{ml}$. For $\mathrm{dib}^{2}, \mathrm{phm}^{E 7}$ mutants or transgenic lines that ubiquitously knock-down dib or phm, fresh embryos were immersed for 5 min in 1× PBS containing $20 \mathrm{E}$ at the final concentration of $0.11 \mathrm{mg} / \mathrm{ml}$ [59]. Survival rates were normalized to the number of embryos used per replicate. Error bars represent standard deviation (data is normally distributed). 


\section{Embryo injection}

PhiC31 constructs were injected at 500-600 ng/ $\mu$ l concentrations. Injections were performed either at the University of Alberta or Da Lat University using standard procedures [115]. In total, 300-500 embryos were injected per construct. Surviving adults were backcrossed to $w^{1118}$ (for Cas13 transgenes) or $y^{2} c h o^{2} v^{1}$ (for crRNA transgenes) and used to generate independent lines.

\section{Quantitative real-time PCR (qPCR)}

Studies were performed as previously described [8, 74]. The extracted RNA (Qiagen RNeasy extraction kit) was reverse-transcribed via the ABI High-Capacity cDNA synthesis kit (Thermo Fisher \#4368814). Synthesized cDNA was used for qPCR (QuantStudio 6 Flex) using KAPA SYBR Fast qPCR master mix \#Sigma KK4601). For each condition, three biological samples were each tested in triplicate. Samples were normalized to rp49 based on the $\Delta \Delta \mathrm{CT}$ method. Error bars represent $95 \%$ confidence intervals.

\section{Statistical analysis}

For the survival studies, survival rates were normalized to the starting number of embryos (50 embryos per replicate). Error bars represent standard deviation (data is normally distributed). In the FREPAIRv2 editing experiment, the editing rate was calculated as the percentage of samples with correct modification out of the total number of sequenced samples. The off-target rate represents the percentage of samples with incorrect modifications out of the total number of sequenced samples. Error bars represent standard deviation (data is normally distributed). In qPCR reactions, samples were normalized to $r p 49$, a housekeeping gene, and based on the $\Delta \Delta C T$ method [116], error bars represent $95 \%$ confidence intervals and contain the error for the calibrator (which is shown without error bars). For multiple comparisons to the same control, we used one-way ANOVA, followed by Dunnett's test. For multiple pair-wise comparisons (in the RNA immunoprecipitation experiments), we applied one-way ANOVA coupled with Tukey's honestly significant difference (HSD) test. At least three biological samples and three technical replicates were analyzed per condition. For quantification of the eCFP signal in immunostains or Western blots, the mean pixel values of the images were analyzed using ImageJ as the corrected total cell fluorescence (CTCF) using the formula: CTCF = selected cell intensity - (area of the chosen cell $\times$ background intensity). The CTCF values were averaged from all biological replicates and normalized to the average CTCF values of no-targeting (NT) crRNA samples. Graphs, standard error calculations, $t$-tests, Dunnett's tests, and Tukey HSD were conducted in Microsoft Excel, SPSS (IBM), and Prism 8 (GraphPad). All data were normally distributed.

\section{Supplementary information}

The online version contains supplementary material available at https://doi.org/10.1186/s13059-020-02193-y.

Additional file 1. Supplementary figures S1-S8

Additional file 2: Table S1. Drosophila codon-optimized Cas13 variants.

Additional file 3. Table S2. List of plasmids.

Additional file 4. Table S3. Primers.

Additional file 5. Table S4. Survival data of all Cas13/crRNAs lines.

Additional file 6. Supplemental methods S1. Cloning procedures for CRISPR/Cas13-crRNA vectors.

Additional file 7. Review history. 


\section{Acknowledgements}

We thank Norbert Perrimon, Simon Bullock, Gerald Rubin, Feng Zhang, Christoph Metzendorf, Kate O'Connor, James D. Sutherland, Andrew Simmonds, and Patrick Hsu for sharing the original plasmids used in this study. Some stocks used in this study were obtained from the Bloomington Drosophila Stock Center (NIH P40ODO18537), the Vienna Drosophila Resource Center, the Japan National Institute of Genetics. This work was supported by the Natural Sciences and Engineering Research Council of Canada (NSERC \#RGPIN-2018-04357) and the Canadian Institute for Health Research (CIHR \#PS 169196)

\section{Peer review information}

Yixin Yao and Kevin Pang were the primary editors on this article and managed its editorial process and peer review in collaboration with the rest of the editorial team.

\section{Review history}

The review history is available as Additional file 7.

\section{Authors' contributions}

N.H. co-designed and carried out most of the experiments and wrote the manuscript. N.D. generated parts of the pC13cr01 plasmids used for cell culture. R.L. assisted with the generation of Cas13 transgenes. K.K.J. acquired funding, supervised trainees, co-designed experiments, and revised the manuscript. The authors read and approved the final manuscript.

\section{Funding}

This work was supported by the Natural Sciences and Engineering Research Council of Canada (NSERC \#RGPIN-201804357) and the Canadian Institute for Health Research (CIHR \#PS 169196).

\section{Availability of data and materials}

The datasets supporting the conclusions of this article are available in the Source Data file at Figshare repository [117]. The pC13cr01 vector collection for cell culture work, p13X and p13B vectors for generating in vivo crRNA transgenes have been deposited at the Drosophila Genetic Resource Center (DGRC) and tentatively scheduled to be available in December 2020. Fly strains carrying CasFB and CasFX will be available at the Bloomington Drosophila Stock Center starting January 2021 as a part of the National Institute of Health project (NIH P40ODO18537). Import permit for cell lines Sg4_CD and Sg4* has been obtained and the live stocks will be sent to DGRC in October 2020. Fly strains, plasmids, and cell lines can also be obtained from our lab upon request.

\section{Ethics approval and consent to participate}

Experiments in this study were conducted in Drosophila cells and live organisms following standard protocols. No human samples were used. No ethics approval is required in this study.

\section{Consent for publication}

All authors participated in this study have been notified about the preparation and submission of the manuscript. Data generated by each author have been collected and used for the preparation of this manuscript.

\section{Competing interests}

The authors declare no competing interests.

Received: 21 May 2020 Accepted: 4 November 2020

Published online: 17 November 2020

\section{References}

1. Makarova KS, Aravind L, Wolf YI, Koonin EV. Unification of Cas protein families and a simple scenario for the origin and evolution of CRISPR-Cas systems. Biol Direct. 2011;6:38.

2. Makarova KS, Wolf YI, Koonin EV. Classification and nomenclature of CRISPR-Cas systems: where from here. CRISPR J. 2018;1:325-36

3. Makarova KS, et al. Evolutionary classification of CRISPR-Cas systems: a burst of class 2 and derived variants. Nat Rev Microbiol. 2020;18:67-83.

4. Makarova KS, et al. An updated evolutionary classification of CRISPR-Cas systems. Nat Rev Microbiol. 2015;13:722-36.

5. van der Oost J, Jore MM, Westra ER, Lundgren M, Brouns SJ. CRISPR-based adaptive and heritable immunity in prokaryotes. Trends Biochem Sci. 2009;34:401-7.

6. Meltzer H, et al. Tissue-specific (ts)CRISPR as an efficient strategy for in vivo screening in Drosophila. Nat Commun. 2019. $10: 2113$.

7. Guilinger JP, Thompson DB, Liu DR. Fusion of catalytically inactive Cas9 to Fokl nuclease improves the specificity of genome modification. Nat Biotechnol. 2014;32:577-82.

8. Huynh N, Zeng J, Liu W, King-Jones K. A Drosophila CRISPR/Cas9 toolkit for conditionally manipulating gene expression in the prothoracic gland as a test case for polytene tissues. G3 (Bethesda). 2018:8:3593-605.

9. Poe AR, et al. Robust CRISPR/Cas9-mediated tissue-specific mutagenesis reveals gene redundancy and perdurance in Drosophila. Genetics. 2019;211:459-72.

10. Port F, Chen HM, Lee T, Bullock SL. Optimized CRISPR/Cas tools for efficient germline and somatic genome engineering in Drosophila. Proc Natl Acad Sci U S A. 2014;111:E2967-76.

11. Xing HL, et al. A CRISPR/Cas9 toolkit for multiplex genome editing in plants. BMC Plant Biol. 2014;14:327.

12. Port F, Bullock SL. Augmenting CRISPR applications in Drosophila with tRNA-flanked sgRNAs. Nat Methods. 2016;13:852-4.

13. Port F, et al. A large-scale resource for tissue-specific CRISPR mutagenesis in Drosophila. bioRxiv:2019636076. 
14. Epinat JC, et al. A novel engineered meganuclease induces homologous recombination in yeast and mammalian cells. Nucleic Acids Res. 2003;31:2952-62.

15. Silva GH, Belfort M, Wende W, Pingoud A. From monomeric to homodimeric endonucleases and back: engineering novel specificity of LAGLIDADG enzymes. J Mol Biol. 2006;361:744-54.

16. Mandell JG, Barbas CF. Zinc finger tools: custom DNA-binding domains for transcription factors and nucleases. Nucleic Acids Res. 2006;34:W516-23.

17. Nakatsukasa T, Shiraishi Y, Negi S, Imanishi M, Futaki S, Sugiura Y. Site-specific DNA cleavage by artificial zinc finger-type nuclease with cerium-binding peptide. Biochem Biophys Res Commun. 2005;330:247-52.

18. Urnov FD, et al. Highly efficient endogenous human gene correction using designed zinc-finger nucleases. Nature. 2005:435:646-51.

19. Joung JK, Sander JD. TALENs: a widely applicable technology for targeted genome editing. Nat Rev Mol Cell Biol. 2013 $14: 49-55$.

20. Miller JC, et al. A TALE nuclease architecture for efficient genome editing. Nat Biotechnol. 2011;29:143-8.

21. Abudayyeh $\mathrm{OO}$, et al. C2c2 is a single-component programmable RNA-guided RNA-targeting CRISPR effector. Science. 2016;353:aaf5573.

22. O'Connell MR. Molecular mechanisms of RNA targeting by Cas13-containing type VI CRISPR-Cas systems. J Mol Biol. 2019:431:66-87.

23. Abudayyeh OO, et al. RNA targeting with CRISPR-Cas13. Nature. 2017;550:280-4.

24. Bassett AR, Tibbit C, Ponting CP, Liu JL. Highly efficient targeted mutagenesis of Drosophila with the CRISPR/Cas9 system. Cell Rep. 2013;4:220-8.

25. Bassett AR, Liu JL. CRISPR/Cas9 and genome editing in Drosophila. J Genet Genomics. 2014;41:7-19.

26. Brown K, Samarsky D. RNAi off-targeting: light at the end of the tunnel. J RNAi Gene Silencing. 2006;2:175-7.

27. Chavez A, et al. Comparison of Cas9 activators in multiple species. Nat Methods. 2016;13:563-7.

28. Perrimon N, Mathey-Prevot B. Matter arising: off-targets and genome-scale RNAi screens in Drosophila. Fly (Austin). 2007;1:1-5.

29. Bellen HJ, et al. The BDGP gene disruption project: single transposon insertions associated with $40 \%$ of Drosophila genes. Genetics. 2004;167:761-81.

30. Bischof J, Björklund M, Furger E, Schertel C, Taipale J, Basler K. A versatile platform for creating a comprehensive UASORFeome library in Drosophila. Development. 2013;140:2434-42.

31. Bischof J, Sheils EM, Björklund M, Basler K. Generation of a transgenic ORFeome library in Drosophila. Nat Protoc. 2014;9: 1607-20.

32. Kennerdell JR, Carthew RW. Heritable gene silencing in Drosophila using double-stranded RNA. Nat Biotechnol. 2000;18: 896-8.

33. Yamamoto $\mathrm{S}$, et al. A drosophila genetic resource of mutants to study mechanisms underlying human genetic diseases. Cell. 2014;159:200-14

34. Gratz SJ, Wildonger J, Harrison MM, O'Connor-Giles KM. CRISPR/Cas9-mediated genome engineering and the promise of designer flies on demand. Fly (Austin). 2013;7:249-55.

35. Gratz SJ, et al. Highly specific and efficient CRISPR/Cas9-catalyzed homology-directed repair in Drosophila. Genetics. 2014;196:961-71.

36. Gratz SJ, Rubinstein CD, Harrison MM, Wildonger J, O'Connor-Giles KM. CRISPR-Cas9 genome editing in Drosophila. Curr Protoc Mol Biol. 2015;111:31.2.1-31.2.20.

37. Lin S, Ewen-Campen B, Ni X, Housden BE, Perrimon N. In vivo transcriptional activation using CRISPR/Cas9 in Drosophila. Genetics. 2015;201:433-42.

38. Ren $X$, et al. Optimized gene editing technology for Drosophila melanogaster using germ line-specific Cas9. Proc Natl Acad Sci U S A. 2013;110:19012-7

39. Xu J, et al. A toolkit of CRISPR-based genome editing systems in Drosophila. J Genet Genomics. 2015;42:141-9.

40. Dominguez AA, Lim WA, Qi LS. Beyond editing: repurposing CRISPR-Cas9 for precision genome regulation and interrogation. Nat Rev Mol Cell Biol. 2016:17:5-15.

41. Gupta RM, Musunuru K. Expanding the genetic editing tool kit: ZFNs, TALENs, and CRISPR-Cas9. J Clin Invest. 2014;124: 4154-61.

42. Port F, et al. A large-scale resource for tissue-specific CRISPR mutagenesis in Drosophila. eLife. 2020;9:e53865.

43. Zirin J, et al. Large-scale transgenic Drosophila resource collections for loss- and gain-of-function studies. Genetics. 2020:214(4):755-67.

44. Ou Q, Zeng J, Yamanaka N, Brakken-Thal C, O'Connor MB, King-Jones K. The insect prothoracic gland as a model for steroid hormone biosynthesis and regulation. Cell Rep. 2016;16:247-62.

45. Danielsen ET, et al. A Drosophila genome-wide screen identifies regulators of steroid hormone production and developmental timing. Dev Cell. 2016:37:558-70.

46. Konermann S, Lotfy P, Brideau NJ, Oki J, Shokhirev MN, Hsu PD. Transcriptome engineering with RNA-targeting type VID CRISPR effectors. Cell. 2018;173:665-676.e14.

47. Abudayyeh $\mathrm{OO}$, et al. A cytosine deaminase for programmable single-base RNA editing. Science. 2019;365:382-6.

48. Abudayyeh OO, Gootenberg JS, Kellner MJ, Zhang F. Nucleic acid detection of plant genes using CRISPR-Cas13. CRISPR J. 2019;2:165-71.

49. Aman R, et al. RNA virus interference via CRISPR/Cas13a system in plants. Genome Biol. 2018;19:1.

50. Cox DBT, et al. RNA editing with CRISPR-Cas13. Science. 2017:358:1019-27.

51. Freije CA, et al. Programmable inhibition and detection of RNA viruses using Cas13. Mol Cell. 2019;76:826-837.e11.

52. Mahas A, Aman R, Mahfouz M. CRISPR-Cas13d mediates robust RNA virus interference in plants. Genome Biol. 2019;20: 263.

53. Smargon AA, et al. Cas13b is a type VI-B CRISPR-associated RNA-guided RNase differentially regulated by accessory proteins Csx27 and Csx28. Mol Cell. 2017;65:618-630.e7.

54. Cherbas $L$, et al. Tools for targeted genome engineering of established Drosophila cell lines. Genetics. 2015;201:1307-18

55. Cherbas $L$, et al. The transcriptional diversity of 25 Drosophila cell lines. Genome Res. 2011;21:301-14. 
56. Laban A, Tobin JF, Curotto de Lafaille MA, Wirth DF. Stable expression of the bacterial neor gene in Leishmania enriettii. Nature. 1990;343:572-4.

57. Lacalle RA, Pulido D, Vara J, Zalacaín M, Jiménez A. Molecular analysis of the pac gene encoding a puromycin N-acetyl transferase from Streptomyces alboniger. Gene. 1989;79:375-80.

58. Lacalle RA, Tercero JA, Jiménez A. Cloning of the complete biosynthetic gene cluster for an aminonucleoside antibiotic, puromycin, and its regulated expression in heterologous hosts. EMBO J. 1992;11:785-92.

59. Huynh N, Wang S, King-Jones K. Spatial and temporal control of gene manipulation in Drosophila via drug-activated Cas9 nucleases. Insect Biochem Mol Biol. 2020;103336.

60. Jia C, et al. New applications of CRISPR/Cas9 system on mutant DNA detection. Gene. 2018;641:55-62.

61. Jiang F, Doudna JA. CRISPR-Cas9 structures and mechanisms. Annu Rev Biophys. 2017:46:505-29.

62. Port F, Muschalik N, Bullock SL. Systematic evaluation of Drosophila CRISPR tools reveals safe and robust alternatives to autonomous gene drives in basic research. G3 (Bethesda). 2015;5:1493-502

63. Wang Q, et al. The CRISPR-Cas13a gene-editing system induces collateral cleavage of RNA in glioma cells. Adv Sci (Weinh). 2019;6:1901299.

64. Caplen NJ, Parrish S, Imani F, Fire A, Morgan RA. Specific inhibition of gene expression by small double-stranded RNAs in invertebrate and vertebrate systems. Proc Natl Acad Sci U S A. 2001;98:9742-7.

65. Elbashir SM, Harborth J, Lendeckel W, Yalcin A, Weber K, Tuschl T. Duplexes of 21-nucleotide RNAs mediate RNA interference in cultured mammalian cells. Nature. 2001;411:494-8.

66. Fire A, Xu S, Montgomery MK, Kostas SA, Driver SE, Mello CC. Potent and specific genetic interference by doublestranded RNA in Caenorhabditis elegans. Nature. 1998;391:806-11.

67. Jackson AL, et al. Expression profiling reveals off-target gene regulation by RNAi. Nat Biotechnol. 2003;21:635-7.

68. Semizarov D, Frost L, Sarthy A, Kroeger P, Halbert DN, Fesik SW. Specificity of short interfering RNA determined through gene expression signatures. Proc Natl Acad Sci U S A. 2003;100:6347-52.

69. Xu D, et al. A CRISPR/Cas13-based approach demonstrates biological relevance of vlinc class of long non-coding RNAs in anticancer drug response. Sci Rep. 2020;10:1794.

70. Yan F, Wang W, Zhang J. CRISPR-Cas12 and Cas13: the lesser known siblings of CRISPR-Cas9. Cell Biol Toxicol. 2019;35: 489-92.

71. Yang LZ, et al. Dynamic imaging of RNA in living cells by CRISPR-Cas13 systems. Mol Cell. 2019;76:981. 997.e7.

72. González-Morales N, Mendoza-Ortíz MÁ, Blowes LM, Missirlis F, Riesgo-Escovar JR. Ferritin is required in multiple tissues during Drosophila melanogaster development. PLoS One. 2015;10:e0133499.

73. Gray NK, Pantopoulos K, Dandekar T, Ackrell BA, Hentze MW. Translational regulation of mammalian and Drosophila citric acid cycle enzymes via iron-responsive elements. Proc Natl Acad Sci U S A. 1996;93:4925-30.

74. Huynh N, Ou Q, Cox P, Lill R, King-Jones K. Glycogen branching enzyme controls cellular iron homeostasis via Iron Regulatory Protein 1 and mitoNEET. Nat Commun. 2019;10:5463.

75. Melefors $\mathrm{O}$. Translational regulation in vivo of the Drosophila melanogaster mRNA encoding succinate dehydrogenase iron protein via iron responsive elements. Biochem Biophys Res Commun. 1996;221:437-41.

76. Surdej P, Richman L, Kühn LC. Differential translational regulation of IRE-containing mRNAs in Drosophila melanogaster by endogenous IRP and a constitutive human IRP1 mutant. Insect Biochem Mol Biol. 2008;38:891-4.

77. Chiaratti MR, et al. Maternal transmission of mitochondrial diseases. Genet Mol Biol. 2020;43:e20190095.

78. Russell O, Turnbull D. Mitochondrial DNA disease-molecular insights and potential routes to a cure. Exp Cell Res. 2014; 325:38-43.

79. Viscomi C, Zeviani M. Strategies for fighting mitochondrial diseases. J Intern Med. 2020;6:665-84

80. Chen Z, et al. Genetic mosaic analysis of a deleterious mitochondrial DNA mutation in Drosophila reveals novel aspects of mitochondrial regulation and function. Mol Biol Cell. 2015;26:674-84.

81. Hill JH, Chen Z, Xu H. Selective propagation of functional mitochondrial DNA during oogenesis restricts the transmission of a deleterious mitochondrial variant. Nat Genet. 2014;46:389-92.

82. $\mathrm{Ma} \mathrm{H}, \mathrm{Xu} \mathrm{H}, \mathrm{O}$ Farrell PH. Transmission of mitochondrial mutations and action of purifying selection in Drosophila melanogaster. Nat Genet. 2014:46:393-7.

83. Jepson JE, Savva YA, Yokose C, Sugden AU, Sahin A, Reenan RA. Engineered alterations in RNA editing modulate complex behavior in Drosophila: regulatory diversity of adenosine deaminase acting on RNA (ADAR) targets. J Biol Chem. 2011;286:8325-37.

84. Niwa R, et al. CYP306A1, a cytochrome P450 enzyme, is essential for ecdysteroid biosynthesis in the prothoracic glands of Bombyx and Drosophila. J Biol Chem. 2004;279:35942-9.

85. Warren JT, et al. Phantom encodes the 25-hydroxylase of Drosophila melanogaster and Bombyx mori: a P450 enzyme critical in ecdysone biosynthesis. Insect Biochem Mol Biol. 2004;34:991-1010.

86. Lind MI, et al. Of two cytosolic aconitases expressed in Drosophila, only one functions as an iron-regulatory protein. J Biol Chem. 2006;281:18707-14.

87. Niwa YS, Niwa R. Transcriptional regulation of insect steroid hormone biosynthesis and its role in controlling timing of molting and metamorphosis. Develop Growth Differ. 2016:58:94-105.

88. Buchman AB, Brogan DJ, Sun R, Yang T, Hsu PD, Akbari OS. Programmable RNA targeting using CasRx in flies. CRISPR J. 2020;3:164-76

89. Gootenberg JS, Abudayyeh OO, Kellner MJ, Joung J, Collins JJ, Zhang F. Multiplexed and portable nucleic acid detection platform with Cas13, Cas12a, and Csm6. Science. 2018;360:439-44.

90. Kellner MJ, Koob JG, Gootenberg JS, Abudayyeh OO, Zhang F. Author correction: SHERLOCK: nucleic acid detection with CRISPR nucleases. Nat Protoc. 2020;15:1311.

91. Metsky HC, Freije CA, Kosoko-Thoroddsen T-SF, Sabeti PC, Myhrvold C. CRISPR-based COVID-19 surveillance using a genomically-comprehensive machine learning approach. bioRxiv. 20202020.02.26.967026.

92. Daniel E, Onwukwe GU, Wierenga RK, Quaggin SE, Vainio SJ, Krause M. ATGme: open-source web application for rare codon identification and custom DNA sequence optimization. BMC Bioinformatics. 2015;16:303.

93. Puigbò P, Guzmán E, Romeu A, Garcia-Vallvé S. OPTIMIZER: a web server for optimizing the codon usage of DNA sequences. Nucleic Acids Res. 2007;35:W126-31. 
94. Behura SK, Severson DW. Codon usage bias: causative factors, quantification methods and genome-wide patterns: with emphasis on insect genomes. Biol Rev Camb Philos Soc. 2013;88:49-61.

95. Nakamura Y, Gojobori T, Ikemura T. Codon usage tabulated from international DNA sequence databases: status for the year 2000. Nucleic Acids Res. 2000;28:292.

96. Vicario S, Moriyama EN, Powell JR. Codon usage in twelve species of Drosophila. BMC Evol Biol. 2007;7:226.

97. Sharp PM, Li WH. The codon adaptation index--a measure of directional synonymous codon usage bias, and its potential applications. Nucleic Acids Res. 1987;15:1281-95.

98. Goetz RM, Fuglsang A. Correlation of codon bias measures with mRNA levels: analysis of transcriptome data from Escherichia coli. Biochem Biophys Res Commun. 2005;327:4-7.

99. Henry I, Sharp PM. Predicting gene expression level from codon usage bias. Mol Biol Evol. 2007;24:10-2.

100. Wright $F$. The 'effective number of codons' used in a gene. Gene. 1990;87:23-9.

101. Gruber AR, Lorenz R, Bernhart SH, Neuböck R, Hofacker IL. The Vienna RNA websuite. Nucleic Acids Res. 2008;36:W70-4.

102. Mathews DH. RNA secondary structure analysis using RNAstructure. Curr Protoc Bioinformatics. 2014;46:12.6.1-25.

103. Reuter JS, Mathews DH. RNAstructure: software for RNA secondary structure prediction and analysis. BMC Bioinformatics. 2010;11:129.

104. Tan Z, Fu Y, Sharma G, Mathews DH. TurboFold II: RNA structural alignment and secondary structure prediction informed by multiple homologs. Nucleic Acids Res. 2017:45:11570-81.

105. Tafer $\mathrm{H}$, et al. The impact of target site accessibility on the design of effective siRNAs. Nat Biotechnol. 2008;26:578-83.

106. Zhu H, Richmond E, Liang C. CRISPR-RT: a web application for designing CRISPR-C2c2 crRNA with improved target specificity. Bioinformatics. 2018;34:117-9.

107. González M, Martín-Ruíz I, Jiménez S, Pirone L, Barrio R, Sutherland JD. Generation of stable Drosophila cell lines using multicistronic vectors. Sci Rep. 2011;1:75.

108. Hadjantonakis AK, Macmaster S, Nagy A. Embryonic stem cells and mice expressing different GFP variants for multiple non-invasive reporter usage within a single animal. BMC Biotechnol. 2002;2:11.

109. Semple Jl, Biondini L, Lehner B. Generating transgenic nematodes by bombardment and antibiotic selection. Nat Methods. 2012;9:118-9.

110. Wang JW, Beck ES, McCabe BD. A modular toolset for recombination transgenesis and neurogenetic analysis of Drosophila. PLoS One. 2012;7:e42102.

111. Gibson DG, Young L, Chuang RY, Venter JC, Hutchison CA, Smith HO. Enzymatic assembly of DNA molecules up to several hundred kilobases. Nat Methods. 2009;6:343-5.

112. Iwaki T, Figuera M, Ploplis VA, Castellino FJ. Rapid selection of Drosophila S2 cells with the puromycin resistance gene. Biotechniques. 2003;35:482-4. 486.

113. Iwaki T, Castellino FJ. A single plasmid transfection that offers a significant advantage associated with puromycin selection in Drosophila Schneider S2 cells expressing heterologous proteins. Cytotechnology. 2008;57:45-9.

114. Liu T, Sims D, Baum B. Parallel RNAi screens across different cell lines identify generic and cell type-specific regulators of actin organization and cell morphology. Genome Biol. 2009;10:R26.

115. Fish MP, Groth AC, Calos MP, Nusse R. Creating transgenic Drosophila by microinjecting the site-specific phiC31 integrase mRNA and a transgene-containing donor plasmid. Nat Protoc. 2007;2:2325-31.

116. Rao X, Huang X, Zhou Z, Lin X. An improvement of the $2^{\wedge}(-$ delta delta CT) method for quantitative real-time polymerase chain reaction data analysis. Biostat Bioinforma Biomath. 2013;3:71-85.

117. Huynh N, Depner N, Larson R, King-Jones K. A versatile toolkit for CRISPR-Cas13-based RNA manipulation in Drosophila. Figshare. 2020. https://figshare.com/s/ec49a9766bffb1bfe712. https://doi.org/10.6084/m9.figshare.12702317.

\section{Publisher's Note}

Springer Nature remains neutral with regard to jurisdictional claims in published maps and institutional affiliations.

Ready to submit your research? Choose BMC and benefit from:
- fast, convenient online submission
- thorough peer review by experienced researchers in your field
- rapid publication on acceptance
- support for research data, including large and complex data types
- gold Open Access which fosters wider collaboration and increased citations
- maximum visibility for your research: over 100M website views per year
At BMC, research is always in progress.
Learn more biomedcentral.com/submissions

